\title{
Spatiotemporal Properties of Sub-Rayleigh and Supershear Rupture Velocity Fields: Theory and Experiments
}

\author{
Michael Mello ${ }^{\mathrm{a}, *}$, Harsha S. Bhat ${ }^{\mathrm{b}}$, Ares J. Rosakis ${ }^{\mathrm{a}}$ \\ ${ }^{a} 1200$ E. California Blvd, Pasadena, CA 91107 \\ ${ }^{b}$ Institut de Physique du Globe de Paris, UMR 7154 CNRS, Paris, France
}

\begin{abstract}
Fundamental spatiotemporal field properties and particle velocity waveform signatures of sub-Rayleigh and supershear ruptures were experimentally investigated through a series of laboratory earthquake experiments. We appeal to dynamic rupture theory to extract and highlight previously unnoticed aspects and results, which are of direct relevance to our new experiments. Kinematic relationships derived from both singular and non-singular solutions are applied to analyze and interpret various features observed in these experiments.

A strong correspondence is demonstrated between particle velocity records obtained in lab experiments and synthetic particle velocity waveform profiles derived from theory. Predicted temporal profiles, sense of particle motion, and amplitude decay properties of sub-Rayleigh and supershear particle velocity waveforms are experimentally verified. In a particular set of supershear rupture experiments, the fault-normal (FN) and fault-parallel (FP) velocity waveforms were simultaneously recorded at fixed, off-fault field points as a shear Mach front swept these locations. Particle velocity records collected over a broad range of stable supershear rupture speeds validate the predicted scaling relationship $\delta u_{1}^{s} / \delta u_{2}^{s}=\sqrt{V_{r}^{2} / C_{s}^{2}-1}=\beta_{s}$, between the FP $\left(\delta \dot{u}_{1}^{s}\right)$ and the FN $\left(\delta \dot{u}_{2}^{s}\right)$ velocity jumps propagated by a shear Mach front. Additional experimental findings include detailed rupture speed measurements of sub-Rayleigh and supershear ruptures and the observation of a supershear daughter crack with vanishing shear Mach front.

Previously unappreciated scaling relations between particle velocity field components, attributed to dilatational and shear waves, are also developed and experimentally verified. In particular, the FP velocity jump $\delta \dot{u}_{1}^{s}\left(x_{1}, x_{2}\right)$ propagated by the shear Mach front, and the sliding speed $\delta \dot{u}_{1}\left(x_{1}, 0^{+}\right)$, measured at a field point positioned extremely close to the frictional fault plane, are shown to obey a speed-dependent scaling relationship given by $\delta \dot{u}_{1}^{s} / \delta \dot{u}_{1}^{+}=1-2 \frac{C_{s}^{2}}{V_{r}^{2}}$, which was gleaned from a non-singular, steady state velocity field solution.
\end{abstract}

${ }^{*}$ Corresponding author
URL: www.http://rosakis.caltech.edu/ (Ares J. Rosakis)

Preprint submitted to Journal of ${ }^{A} T_{E} X$ Templates

February 25, 2016 
Keywords: vibrometer, spatiotemporal, sub-Rayleigh, supershear laboratory earthquake experiment (LEQ), fault-normal, fault-parallel, dilatational field, shear Mach front

\section{Introduction}

There have been some seminal papers published over the past half century, which have all contributed to the current theoretical understanding of dynamic mode II ruptures and the advancement of earthquake rupture models. The

5 earliest theoretical models trace back to the analysis of a steady state semiinfinite crack, subjected to a combined mode I and mode II loading by Craggs (1960) and Kostrov (1964), and a self-similar solution for a propagating mode II shear crack given by Kostrov (1964). Each of these solutions is characterized by a singular crack front, with surrounding elastic fields, which decay as the

10 inverse square-root of the radial distance from the crack front. Craggs (1960) realized that stable crack growth results as energy from the surrounding linear elastic field is drawn into the singular crack tip. Such a condition can only arise if the rupture propagates at speeds below the Rayleigh wave speed $\left(C_{R}\right)$ of the surrounding medium, i.e., $\left(V<C_{R}\right)$. Kostrov (1964) recognized that 15 energy must radiate out from the crack tip if the rupture speed lies between the Rayleigh wave speed and shear wave speed $\left(C_{s}\right)$ of the surrounding linear elastic medium, i.e., $\left(C_{R}<V_{r}<C_{s}\right)$. The latter scenario is rejected on physical grounds thus implying that a mode II shear crack will tend to propagate in the sub-Rayleigh rupture speed regime $\left(V_{r}<C_{R}\right)$, Kostrov, 1964).

${ }_{20}$ Burridge (1973) was the first to propose conditions under which a plainstrain, self-similar mode II crack can bypass the forbidden rupture speed regime $\left(C_{R}<V_{r}<C_{s}\right)$ and propagate within the intersonic rupture speed regime $\left(C_{s} \leq V_{r} \leq C_{d}\right)$, where $\left(C_{d}\right)$ represents the dilatational wave speed. A simple Coulomb friction relation was assumed to model the interfacial shear strength

25 ahead of the primary (sub-Rayleigh) crack front. The approach assured that stresses would remain bounded at the primary crack front but failed to account for the effects of inelastic (cohesive) energy losses at the singular crack tip. In spite of this limitation, the analysis identified the presence of a peak in the shear stress field, which propagates ahead of the primary crack front at the shear 30 wave speed. This was a key finding, which revealed a natural mechanism for nucleating a secondary (intersonic ) rupture ahead of the primary crack front if the magnitude of the peak shear stress exceeded the intrinsic fault strength. The analysis also concluded that the intersonic crack speed would rapidly approach the dilatational wave speed $\left(C_{d}\right)$ of the surrounding medium.

35 Andrews (1976) produced the very first numerical finite difference calculations, which successfully simulated a sub-Rayleigh to supershear rupture transition. The model featured a linear slip-weakening failure criterion, which defined the loss of frictional strength with increased slip over a characteristic length along the fault plane. The implementation of a finite slip-weakening distance 
40 eliminated the crack tip singularity and provided an energy dissipation mechanism, which was lacking in the earlier analysis by Burridge (1973). Numerical trials revealed a peak in the shear stress field positioned just ahead of the primary (sub-Rayleigh) crack front, as noted by Burridge (1973), which nucleated a secondary crack when the local fault strength was exceeded. The trailing 45 edge of a newly spawned secondary shear crack was observed to merge rapidly with the primary (trailing) sub-Rayleigh rupture while the leading edge raced ahead as an intersonic crack front. A key outcome of this study was the identification of a mechanically stable portion of the intersonic rupture speed regime $\sqrt{2} C_{s} \leq V_{R} \leq C_{d}$, commonly referred to as the supershear rupture speed do-

Burridge et al. (1979) revisited the problem of admissible rupture speeds by conducting a rigorous stability analysis of a steady-state shear crack driven by a point load maintained at a constant distance behind the crack tip. Energy dissipation was accounted for in the analysis through a Dugdale-type cohesive traction relation confined to a small region around the crack tip. Conclusions regarding the stability of intersonic ruptures were in general agreement with the numerically based findings of Andrews (1976). Specifically, (1) the intersonic velocity regime $\sqrt{2} C_{s} \leq V_{R} \leq C_{d}$ was identified to be stable with a unique local minima within this velocity domain and (2) the intersonic velocity regime

${ }_{60} C_{s} \leq V_{R}<\sqrt{2} C_{s}$ was shown to be unstable. Conclusions regarding the stability of sub-shear crack propagation were also consistent with the earlier findings by Craggs (1960) and Kostrov (1964).

Ongoing work during this same period by Fossum and Freund (1975) and Freund (1979) was directed toward the interpretation of crustal ruptures associated 65 with natural earthquakes. The seminal work by Freund (1979) provided closedform analytical solutions for the singular elastic stress and particle velocity fields radiated by a 2D steady-state shear crack. Sub-Rayleigh and intersonic crack field solutions were derived for the shear stress $\left(\sigma_{12}\right)$ and fault-parallel velocity component $\left(\dot{u}_{1}\right)$. The amplitude of the resulting field expressions is modulated

70 by a velocity-dependent "dynamic stress intensity factor", which characterizes the intrinsic strength of the fracture plane. Despite the singular nature of the steady state dynamic shear crack model and the glaring lack of an intrinsic length scale, the stress and particle velocity field solutions embody fundamental traits of a propagating mode II rupture, which are highly characteristic of 75 natural earthquake ruptures.

Freund (1979) also examined the stability of sub-shear crack propagation by considering the relationship between the normalized stress intensity factor and the crack tip speed. Results were consistent with those of Burridge et al. (1979), along with the earlier stability analysis conducted by Fossum and Freund (1975), shich considered the energy flux into a dynamic shear crack. The stability of intersonic ruptures was also briefly addressed although no corresponding analysis was provided.

More realistic rupture models (either crack-like or pulse-like) should feature finite shear cohesive zones at the rupture fronts as opposed to singularities, ${ }_{85}$ which imply infinite stress at the crack tip. The physical dimension of the 
cohesive zone also introduces a natural length scale, which is otherwise lacking in the singular elastic model. In the earliest earthquake rupture models, the rupture pulse was modeled as a propagating dislocation. Aki and Richards (1968) successfully applied this approach to the interpretation of near-source ground motion records gathered during the 1966 Parkfield earthquake. The subRayleigh dislocation model by Aki and Richards (1968) was generalized to the case of any arbitrary shear traction by Broberg (1978) and then later extended to the case of intersonic rupture pulses (Broberg, 1989). Broberg's solutions focused upon the on-fault and near-source properties of radiated rupture fields vanishingly small breakdown zone. Broberg (1994) followed with an analysis of intersonic ruptures within the context of self-similar expanding shear cracks with a cohesive zone. Dunham and Archuleta (2005) subsequently extended the earlier solution by Broberg (1989), to obtain a non-singular analytical solution for the stress and particle velocity fields radiated by a $2 \mathrm{D}$ steady-state intersonic slip-pulse, subject to a prescribed shear traction within a finite slip zone.

\section{Supershear Earthquake Ruptures}

The past two decades have witnessed a veritable explosion of publications related to the theory of supershear earthquake ruptures. Increased attention to this topic was partly motivated by the observations of supershear ruptures in the original dynamic shear impact studies conducted by Lambros and Rosakis (1995) and in subsequent investigations using the "laboratory earthquake experiment" (LEQ) developed by Xia et al. (2004, 2005). Recent experimental work in this area by other research groups includes laboratory observations of supershear

${ }_{110}$ ruptures during stick-slip experiments on Westerly granite under high applied normal stress (F.X.Passelegue et al., 2013) and sliding friction investigations by Latour et al. (2011) and Svetlizky and Fineberg (2014).

Increased attention on this topic was also spurred on by an ever-growing list of reported supershear earthquake ruptures, where the crustal rupture speed was inferred to have exceeded the local shear wave speed along an identified segment of the ruptured fault. In all cases, rupture speed estimates have been confined to the stable (supershear) rupture speed domain $\left(\sqrt{2} C_{s} \leq V_{R} \leq C_{d}\right)$. Supershear earthquakes have also generally been large-magnitude events, which occurred on long and relatively straight strike-slip faults (Robinson et al., 2006).

${ }_{120}$ Three of the most notable supershear earthquake events were the $1999 M_{w} 7.6$ (Izmit,Turkey) earthquake (Bouchon et al., 2001, 2002, Bouchon and Vallee, 2003 Bouin et al., 2004), the $2001 M_{w} 7.8$ (Kokoxili, Tibet) earthquake along the Kunlun fault (Bhat et al. 2007), and the $2002 M_{w} 7.9$ (Denali, Alaska) earthquake (Ellsworth et al., 2004| Dunham and Archuleta, 2004).

Theoretical and numerical models predict that a supershear rupture should be characterized by a fault parallel particle velocity component, which dominates in magnitude over the fault normal component. This feature is in direct contrast the ground motion waveforms resulting from sub-Rayleigh earthquake 
ruptures, where the fault-normal velocity component dominates (Freund, 1979

130 Aagaard and Heaton 2004 Dunham and Archuleta, 2004. 2005).

The $2002 M_{w} 7.9$ Denali earthquake was an exceptionally well-characterized event due to an array of well-positioned, near-field ground motion stations positioned along the Trans-Alaska Pipeline System (TAPS). Well-characterized ground motion records acquired at Pump Station 10 (PS10), located $\approx 3 \mathrm{~km}$ 135 north of the Denali strike-sip fault, bear the distinct kinematic signatures of a leading supershear rupture pulse, followed by a (secondary) sub-Rayleigh rupture. Dunham and Archuleta (2004) applied a spontaneous dynamic rupture propagation model with a slip-weakening friction law and built-in healing mechanism, which simulated the supershear and trailing secondary (sub-Rayleigh) rup140 ture pulses observed in the PS10 ground motion records. Scaled particle velocity waveforms, which capture the PS10 supershear and trailing sub-Rayleigh waveform signatures were also experimentally demonstrated by Mello et al. (2014) using a modified LEQ specimen configuration, which featured a partially locked frictional fault.

Theoretical and numerical investigations of supershear ruptures in 2D conducted by Dunham and Archuleta (2004); Bhat et al. (2007) and in 3D by Dunham and Bhat (2008) also predict that ground motion due to the passage of the shear Mach front is virtually unattenuated at large distances from the fault. In 2D steady-state supershear rupture models, the shear Mach front carries ground motion unattenuated, out to infinity. In reality, the amplitude of a radiated shear Mach front is expected to decay as $1 / \sqrt{r}$ due to spatial dispersion of the expanding $\mathrm{S}$ waves, which coherently interfere to form the expanding Mach front. By similar reasoning, one should expect the field strength of a 3D shear Mach cone to decay as $1 / r$ due to the geometric dispersion of radiated 155 spherical S waves. Bhat et al. (2007) reasoned that the velocity field carried by the shear Mach front should, at least, be sustained out to distances comparable to the depth of the seismogenic zone. Dunham and Bhat (2008) followed with a 3D numerical model, which demonstrated the effects of geometric dispersion while also confirming the earlier estimation by Bhat et al. (2007). Theoretical 160 attenuation estimates of supershear ground motion are partially supported by field observations of anomalous ground cracks observed several kilometers from the main fault following the 2001 Kokoxili (Kunlun) $M_{w} 7.8$ event in Tibet (Bhat et al., 2007). Clustering of aftershocks at off-fault distances comparable to the depth of the seismogenic zone was also observed by Bouchon and Karabulut (2008), following the 1999 moment magnitude, $M_{w} 7.6$ Izmit earthquake.

\section{Steady State Interpretation of Laboratory Earthquake Data}

In the present paper, we present findings from a series of laboratory earthquake experiments (LEQ) designed to investigate and systematically probe the velocity waveform signatures, attenuation characteristics, and spatiotemporal 170 field properties of sub-Rayleigh and supershear ruptures. The dynamic ruptures generated in our LEQ experiments are predominantly $2 \mathrm{D}$ in character since they are constrained to propagate along the straight frictional fault formed by two 
compressed, $10 \mathrm{~mm}$ thick, trapezoidal plates of a Homalite-100 laboratory test specimen. Just as in nature, the ruptures observed in our experiments also tend to propagate in a self-similar way when viewed from a "large distance" from the fault plane. However, all measurements presented in this paper have been concentrated at very small fault-normal distances $\left(\xi_{2}\right)$ from the fault of length $(L=174 \mathrm{~mm})$. As such, the vast majority of these experiments correspond to $\left(0<\xi_{2} / L<0.14\right)$ with the upper bound corresponding to $\xi_{2}=24 \mathrm{~mm}$ in only 180 one particular experiment. It is the very fact that we are conducting near fault measurements that make steady state theories very relevant for comparison to our experiments. Indeed, as $\xi_{2} / L \rightarrow 0$ the steady state approximation becomes increasingly accurate as demonstrated by Liu and Rosakis (1994) and Freund (1998).

185 Liu and Rosakis (1994) in particular, considered the case of a randomly transient, mixed-mode rupture propagating at random sub-Rayleigh speeds along a randomly curved path under general transient loading. Self-similar shear ruptures propagating along a straight path with constant rupture speeds are a special transient case covered by this study. The study by Liu and Rosakis 190 (1994) scrutinizes the higher order asymptotic structure of the growing rupture tip as a function of the radial distance from the tip. Results of this study clearly show that very near the rupture tip the asymptotically dominant terms, which govern the rupture process, have the same radial and angular nature as those predicted by the equivalent steady state asymptotic analysis. Specifically, the ${ }_{195} 1 / \sqrt{r}$ structure is recovered, while the predicted rupture speed functional dependence is also recovered. The only difference is that the amplitudes of the dominant field terms, as well as the rupture speed, now vary with time, i.e., $K=K_{I I}(t), V_{r}=V_{r}(t)$ in the transient problem. It is only when the distance from the rupture tip is increased that transient higher order terms change the actual nature of the field structure. During supershear rupture events, the presence of characteristics in the form of shear Mach cones is expected to radiate the local steady state nature of the shear field solution even further from the fault plane. The remarkable agreement between our near-fault particle velocity records and the steady state rupture theories strongly support the above con-

205 clusions, most notably for off-fault field point measurements conducted at FN distances $\xi_{2} \leq L / 10$ from the fault plane.

Bearing these points in mind, we appeal to $2 \mathrm{D}$ steady state dynamic rupture theory and extract previously unnoticed aspects and results of relevance to our experimental findings. Kinematic relationships gleaned from both singular and non-singular solutions are used as tools for analyzing and interpreting various aspects of our experimental observations. In particular, we revisit the 2D singular elastic, steady state dynamic shear crack model by Freund (1979, 1998) and highlight spatiotemporal properties and kinematic scaling relationships obtained from this solution, which are observed in LEQ experiments. We also examine 215 and expand upon the non-singular 2D steady-state intersonic slip pulse model by Dunham and Archuleta (2005), and present a scaling relationship, gleaned from this model, which relates the rupture sliding speed at the fault plane with the fault-parallel velocity jump propagated by the shear Mach front. 


\section{Spatiotemporal Field Properties and Scaling Relations Gleaned from the 2D Steady-State Singular Elastic Shear Crack Model}

4.1. Overview of the 2D Steady State, Singular Elastic Solution for a Dynamic Shear Crack: Sub-Rayleigh Rupture Speed Regime $\left(V_{r}<C_{R}\right)$

Freund (1979, 1998) rigorously solved the 2D plane strain problem of a steady state dynamic (mode II) shear crack in a linear elastic half-space. The problem 225 was posed under the assumption of plane strain, with the crack front oriented parallel to the $x_{3}$ axis of a Cartesian coordinate frame. A right-lateral/righttraveling shear crack was assumed to propagate along the $x_{1}$ axis in the global coordinate system, with crack-tip coordinates $\left(x_{1}=l(t), 0\right)$ and instantaneous crack tip speed $V_{r}(t)=\dot{l}(t)$, where $l(t)$ is a continuous function of time. A local coordinate system $\left(\xi_{1}, \xi_{2}\right)$ was then introduced with the origin selected to coincide with the crack tip, such that $\xi_{1}=x_{1}-l(t)$ and $\xi_{2}=x_{2}$. A local polar coordinate system $(r, \theta)$ was also introduced, whereby $r=\sqrt{\xi_{1}^{2}+\xi_{2}^{2}}$ and $\theta=\tan ^{-1}\left(\xi_{2} / \xi_{1}\right)$. The derivation leads to a standard Hilbert problem in analytic function theory which is then solved subject to the prescribed traction-free boundary conditions on the crack surfaces.

The sub-Rayleigh singular elastic (asymptotic) solutions for the (in-plane) particle velocity field components $\dot{u}_{1}(r, \theta)$ and $\dot{u}_{2}(r, \theta)$ of a right-lateral/righttraveling, mode II shear crack are given by

$$
\dot{u}_{1}=\frac{V_{r} \alpha_{s} K_{I I}(t)}{\mu D \sqrt{2 \pi r}}\left[2 \frac{\sin \frac{1}{2} \theta_{d}}{\sqrt{\gamma_{d}}}-\left(1+\alpha_{s}^{2}\right) \frac{\sin \frac{1}{2} \theta_{s}}{\sqrt{\gamma_{s}}}\right]
$$

and

$$
\dot{u}_{2}=-\frac{V_{r} K_{I I}(t)}{\mu D \sqrt{2 \pi r}}\left[2 \alpha_{d} \alpha_{s} \frac{\cos \frac{1}{2} \theta_{d}}{\sqrt{\gamma_{d}}}-\left(1+\alpha_{s}^{2}\right) \frac{\cos \frac{1}{2} \theta_{s}}{\sqrt{\gamma_{s}}}\right] .
$$

240 Here, $K_{I I}(t)$ is the instantaneous mode II dynamic stress intensity factor, $\mu$ represents the shear modulus and the factors $\alpha_{s}, \alpha_{d}, \gamma_{s}, \gamma_{d}, \theta_{s}$, and $\theta_{d}$ are given by

$$
\begin{gathered}
\theta_{s}=\tan ^{-1}\left(\alpha_{s} \tan \theta\right) ; \quad \theta_{d}=\tan ^{-1}\left(\alpha_{d} \tan \theta\right) \\
\gamma_{s}=\sqrt{1-\left(V_{r} \sin \theta / C_{s}\right)^{2}} ; \quad \gamma_{d}=\sqrt{1-\left(V_{r} \sin \theta / C_{d}\right)^{2}} \\
\alpha_{s}=\sqrt{1-\left(V_{r} / C_{s}\right)^{2}} ; \quad \alpha_{d}=\sqrt{1-\left(V_{r} / C_{d}\right)^{2}} .
\end{gathered}
$$

${ }_{245}$ The factor $D$ in the denominator of Eqs. 1, 2 is the Rayleigh wave function given by

$$
D=4 \alpha_{s} \alpha_{d}-\left(1+\alpha_{s}^{2}\right)^{2} .
$$

The root of Eq. (6) corresponds to the Rayleigh wave speed over a homogeneous, linear elastic half-space Graff (1991). Careful examination of Eq. (6) reveals that $D \rightarrow 0$ as $V_{r} \rightarrow C_{R}$, while $D>0$ corresponds to admissible sub-Rayleigh

250 rupture speeds $V_{r}<C_{R}$, and $D<0$, corresponds to the forbidden rupture speed domain $C_{R}<V_{r}<C_{s}$. We note as well, how $D<0$ implies that the sign of each motion component given by Eqs. 12 would both reverse if the forbidden rupture speeds $C_{R}<V_{r}<C_{s}$ were admissible. 


\subsection{Spatiotemporal Field Properties and Particle Velocity Waveform Signatures} of a $2 D$ Steady State Sub-Rayleigh Shear Crack: $\left(V_{r}<C_{R}\right)$

The particle velocity field expressions given by Eqs. (1) and (2) apply to a right-lateral/right-traveling $(r l / r t)$ shear crack. The expressions were adjusted to yield the solution for a right-lateral/left-traveling $(r l / l t)$ shear crack in order to match the rupture propagation direction and sense of particle motion observed in the LEQ experiments reported in this paper. The adjusted particle velocity field solutions for a $(r l / l t)$ shear crack are easily obtained by invoking $\left(u_{2}\right)_{r l / l t}=-\dot{u}_{2 r l / r t}$ and $(\theta)_{(r l / l t)}=-(\theta)_{r l / r t}$. The particle velocity vector field plot and synthetic particle velocity waveforms depicted in Figure 1 correspond to a $(r l / l t)$ sub-Rayleigh shear crack. The plots were generated using a convenient and arbitrary choice of values for the material constants corresponding to $K_{I I} / \mu=0.2 \mathrm{~mm}^{1 / 2}, c_{s}=1.3 \mathrm{~mm} / \mu \mathrm{s}$, and $c_{d}=2.6 \mathrm{~mm} / \mu \mathrm{s}$ along with an arbitrary $50 \mathrm{~mm} \times 50 \mathrm{~mm}$ field corresponding to a $2000 \times 2000$ point grid.

Figure 1(a) represents the velocity vector field $\dot{\vec{u}}\left(\xi_{1}, \xi_{2}\right)$ of a sub-Rayleigh shear crack propagating at $V_{r}=0.9 C_{s}$. The dashed black horizontal line in the figure represents the fault plane $\left(\xi_{2}=0\right)$. The colors displayed by the plot legend correspond to the magnitude of the particle velocity $\left(|\dot{\vec{u}}|=\sqrt{\dot{u}_{1}^{2}+\dot{u}_{2}^{2}}\right)$, while the white arrows of varying length represent the relative magnitude and direction of the particle velocity vectors. The velocity field is characterized by a dominant fault-normal particle motion component $\left(\dot{u}_{2}\right)$, as revealed by the steeply inclined and downward directed velocity vectors within the half-space $\xi_{1}<0$.

The curve displayed in Figure 1(b) depicts the normalized amplitude decay of the FN particle velocity component plotted versus the normalized distance $\left(\xi_{2} / \xi_{2}^{\max }\right)$. The horizontal axis is normalized with respect to the maximum off-

280 fault distance $\left(\xi_{2}^{\max }=25 \mathrm{~mm}\right)$, which was assumed in the analysis. The amplitude decay curve was generated by computing the ratio $\left.\delta \dot{u}_{2}\left(\xi_{1}, \xi_{2}\right)\right) / \delta \dot{u}_{2}\left(\xi_{1}, 0^{+}\right)$, where $\delta \dot{u}_{2}\left(\xi_{1}, \xi_{2}\right)$ represents the amplitude of the maximum velocity swing occurring along $\xi_{2}=c$, and $\delta \dot{u}_{2}\left(\xi_{1}, 0^{+}\right)$is the amplitude of the velocity jump at a "near-fault" point $\left(\xi_{1}, 0^{+}\right)$. The same amplitude decay curve could have been generated by conducting a similar analysis of the FP component $\left(\dot{u}_{1}\right)$. The near field point $\left(\xi_{1}, 0^{+}\right)$was carefully selected in order to exclude the crack tip singularity. In this case $\xi_{2} \rightarrow 0^{+}$corresponds to a fault-normal distance $\xi_{2}=0.2 \mathrm{~mm}$ within the arbitrarily imposed length scale. The curve in Figure 1 (b) depicts an amplitude decay, which is proportional to $1 / \sqrt{\xi_{2} / \xi_{2}^{\max }}$ in accor-

290 dance with Eqs. (1) and (2). Figures 1(c,d) represent synthetic particle velocity waveforms corresponding to the fault-parallel $\left(\dot{u}_{1}\right)$ and fault-normal $\left(\dot{u}_{2}\right)$ motion components, computed at various distances $\left(\xi_{2}\right)$ from the fault plane. Line plots were extracted from the adjusted particle velocity field expressions for a $(r l / l t)$ rupture and replotted with an equivalent time axis $\left(t=\xi_{1} / V_{r}\right)$ in accordance with the steady state assumption. The curves plotted in red highlight the "near-fault" velocity waveforms while the blue curves correspond to particle velocity waveforms plotted in fault-normal distance increments of $\Delta \xi_{2}=2 \mathrm{~mm}$, plotted out to a distance $\xi_{2}=6 \mathrm{~mm}$ within the arbitrarily defined length scale. 
(a)

(c)

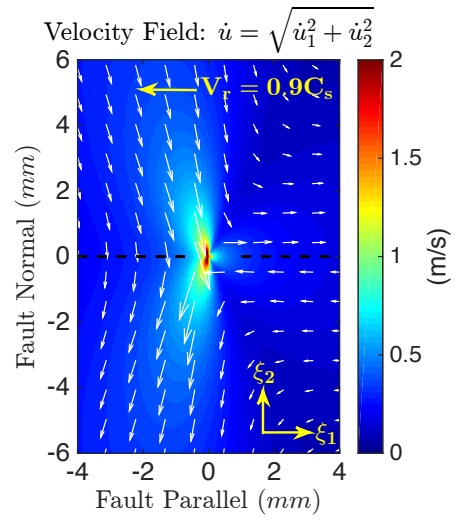

Fault Normal Particle Velocity Waveforms

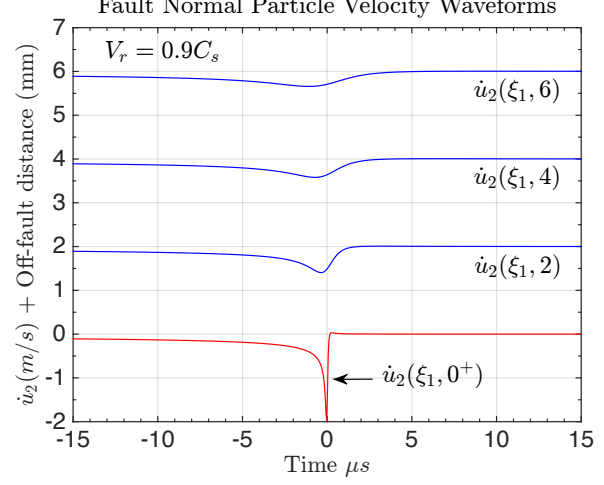

(b) Sub-Rayleigh Velocity Field Decay

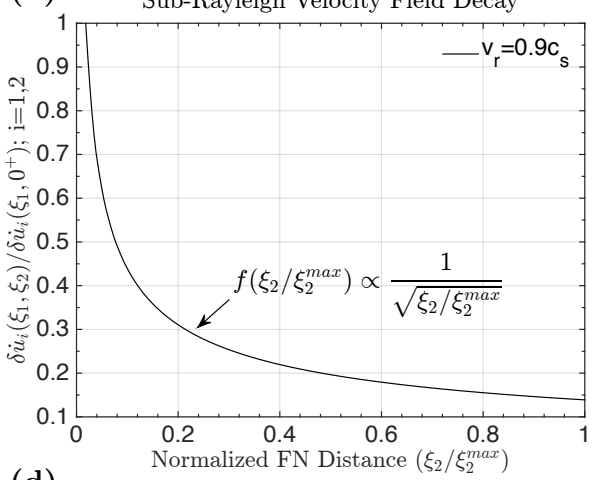

(d)

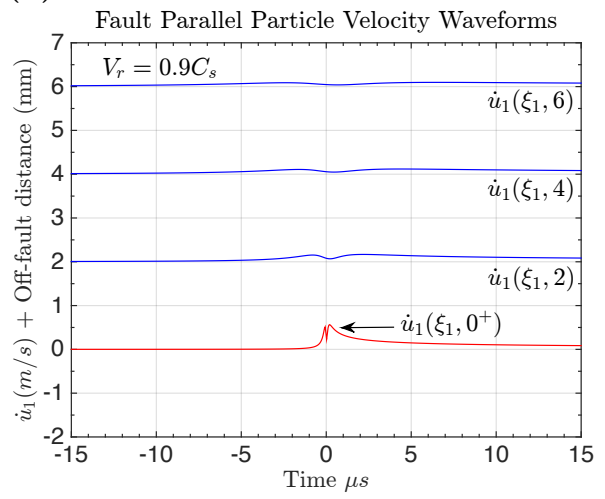

Figure 1: (a) Sub-Rayleigh velocity field plot for a right-lateral/left-traveling shear crack. (b) Normalized amplitude decay of the sub-Rayleigh velocity field components. (c) Synthetic FN velocity waveforms at incremented distances from the fault. (d) Synthetic FP velocity waveforms at incremented distances from the fault. Note: The crack tip singularity was excluded in Figures (c,d) by normalizing with respect to the particle velocity $\dot{u}_{i}\left(\xi_{1}, 0^{+}\right)$, where $\xi_{2}=0^{+}$is equivalent to $\xi_{2}=0.2 \mathrm{~mm}$ within the arbitrarily imposed length scale.

Two distinct and fundamental spatiotemporal properties of a steady state (subRayleigh) rupture velocity field are revealed: (1) the particle velocity waveforms are characterized by a prominent fault-normal component, as previously noted, which clearly dominates in magnitude over the fault-parallel component, and (2) sharp velocity waveforms at the fault plane are very quickly stripped of their frequency content and undergo a rapid amplitude decay with increased faultnormal distance $\left(\xi_{2}\right)$, as described by the normalized attenuation plot in Figure 1 (b).

In a later section of this paper a strong correspondence is demonstrated between sub-Rayleigh particle velocity records obtained in LEQ experiments and the general profile and predicted sense of particle motion of the synthetic waveforms depicted in Figures 1(c,d). 
4.3. Overview of the 2D Steady State, Singular Elastic Solution for a Dynamic Shear Crack : Intersonic Rupture Speed Regime $\left(C_{s} \leq V_{r} \leq C_{d}\right)$

Freund $(1979,1998)$ also considered the problem for the stress and particle velocity fields radiated by a steady-state shear crack propagating in the intersonic rupture speed domain $\left(C_{s} \leq V_{r} \leq C_{d}\right)$. The problem was posed under the assumption of plane strain with traction-free boundary conditions imposed on the crack faces. A right-lateral/right-traveling shear crack was assumed to propagate along the $x_{1}$ direction, with the crack front oriented parallel to the $x_{3}$ axis of a global Cartesian coordinate frame. A Cartesian coordinate frame $\left(\xi_{1}, \xi_{2}\right)$ is again introduced, with its origin at the crack tip, which is equivalently expressed in polar form $(r, \theta)$, whereby $r=\sqrt{\xi_{1}^{2}+\xi_{2}^{2}}$ and $\theta=\tan ^{-1}\left(\xi_{2} / \xi_{1}\right)$.

The derivation leads to a standard Hilbert problem in analytic function theory which is then solved subject to the prescribed traction-free boundary conditions on the crack surfaces. The intersonic, singular elastic (asymptotic) so325 lutions for the (in-plane) particle velocity field components $\dot{u}_{1}(r, \theta)$ and $\dot{u}_{2}(r, \theta)$ corresponding to a right-lateral/right-traveling, mode II shear crack are given by

$$
\dot{u}_{i}=A V_{r}\left(\dot{u}_{i}^{s}+\dot{u}_{i}^{d}\right) ; i=1,2
$$

where $A=K_{I I} / \mu$ represents an effective dynamic stress intensity factor, as previously defined, and $V_{r}$ is the rupture speed $\left(C_{s} \leq V_{r} \leq C_{d}\right)$.

The shear field contributions of Eq. (7) are given by

$$
\dot{u}_{1}^{s}=-\operatorname{sgn}\left(\xi_{2}\right) \frac{\left(2-\frac{V_{r}^{2}}{C_{s}^{2}}\right) \sin (\pi q)}{2\left(-\xi_{1}-\beta_{s}\left|\xi_{2}\right|\right)^{q}} H\left(-\xi_{1}-\beta_{s}\left|\xi_{2}\right|\right)
$$

and

$$
\dot{u}_{2}^{s}=\frac{\left(2-\frac{V_{r}^{2}}{C_{s}^{2}}\right) \sin (\pi q)}{2 \beta_{s}\left(-\xi_{1}-\beta_{s}\left|\xi_{2}\right|\right)^{q}} H\left(-\xi_{1}-\beta_{s}\left|\xi_{2}\right|\right)
$$

where the variables $\beta_{s}$ and $q$ are given by

$$
\begin{gathered}
\beta_{s}=\sqrt{\left(V_{r} / C_{s}\right)^{2}-1} \\
q=\frac{1}{\pi} \tan ^{-1}\left[\frac{4 \alpha_{d} \beta_{s}}{\left(2-V_{r}^{2} / C_{s}^{2}\right)^{2}}\right]
\end{gathered}
$$

and $\operatorname{sgn}\left(\xi_{2}\right)$ represents the sign function, which is equal to +1 for $\xi_{2}>0$ and -1 for $\xi_{2}<0$. Eqs. (8) and (9) represent the velocity components propagated by the shear Mach fronts which extend from the rupture tip and envelope the radiated shear field.

The dilatational velocity field components in Eq. 7 are given by

$$
\dot{u}_{1}^{d}=\frac{\sin \left(q \theta_{d}\right)}{r_{d}^{q}} ; \quad \dot{u}_{2}^{d}=-\alpha_{d} \frac{\cos \left(q \theta_{d}\right)}{r_{d}^{q}}
$$


where the variable $r_{d}$ is given by

$$
r_{d}=\sqrt{\xi_{1}^{2}+\left(\alpha_{d} \xi_{2}\right)^{2}}
$$

with $\theta_{d}, \alpha_{d}$ given by Eq. (3) and Eq. (5), respectively. The forms presented here for $\dot{u}_{2}^{d}$ and $\dot{u}_{2}^{s}$ correct a minor typographical error in equation 4.3.31 in Freund (1998).

Examination of Eq. 11 reveals that that $0 \leq q \leq 1 / 2$. A maximum value $q=1 / 2$ is approached as $V_{r} \rightarrow \sqrt{2} C_{s}$ whereas $q \rightarrow 0$ as $V \rightarrow C_{s}$ or $V_{r} \rightarrow C_{d}$.

345 The singularity $r_{d}^{-q}$ is thus generally weaker than the $r^{-1 / 2}$ singularity, which implies that the dilatational field will tend to decay more slowly compared to the fields radiated by a sub-Rayleigh rupture field. Interestingly, the $r^{-1 / 2}$ singularity remerges only in the limiting case $V_{r} \rightarrow \sqrt{2} C_{s}$, corresponding to the lower bound of the supershear rupture speed regime.

4.4. Spatiotemporal Properties of a Supershear Crack Velocity Field: 2D Steady State, Singular Elastic Solution $\left(\sqrt{2} C_{s} \leq V_{r}<C_{d}\right)$

The particle velocity vector field plot and synthetic particle velocity waveforms depicted in Figure 2 correspond to a right-lateral/left-traveling $(\mathrm{rl} / \mathrm{lt})$ supershear shear crack. The adjusted particle velocity field solutions were obtained by invoking $\left({\dot{u_{2}}}^{s}\right)_{r l / l t}=-\left({\dot{u_{2}}}^{s}\right)_{r l / r t},(\theta)_{(r l / l t)}=-(\theta)_{r l / r t}$, and adjusting the characteristic equation from $\left(-\xi_{1}-\beta_{s}\left|\xi_{2}\right|\right)$, for the (rl/rt) rupture to $\left(\xi_{1}-\beta_{s}\left|\xi_{2}\right|\right)$ for the (rl/lt) rupture. The plots were generated using a convenient and arbitrary choice of values for the material constants corresponding to $A=0.45 \mathrm{~mm}^{1 / 2}, c_{s}=1.3 \mathrm{~mm} / \mu \mathrm{s}$, and $c_{d}=2.6 \mathrm{~mm} / \mu \mathrm{s}$, along with an arbitrary $50 \mathrm{~mm} \times 50 \mathrm{~mm}$ field constructed of a $2000 \times 2000$ point grid.

Figure 2(a) displays the particle velocity field $\dot{\vec{u}}\left(\xi_{1}, \xi_{2}\right)$ of a right-lateral/lefttraveling supershear crack propagating with rupture speed $V_{r}=1.6 C_{s}$. The supershear rupture tip is enveloped within an elliptically-shaped field lobe, characterized by a velocity field which circulates about the rupture tip. A prominent shear Mach cone extends from the rupture tip and bounds the radiated shear field. The velocity field, which extends beyond the shear Mach fronts, is thus exclusively dilatational since all shear radiation emitted by the rupture is confined, by default, within the region bounded by the shear Mach cone. The leading concentrated field lobe is henceforth referred to as the "dilatational field lobe". The velocity vector field is characterized by a dominant fault-parallel particle velocity component $\left(\dot{u}_{1}\right)$ as revealed by the relatively shallow inclination $(\theta<\pi / 4)$ of the velocity field vectors both within the dilatational field lobe and along the shear Mach fronts.

Figure 2(b) represents the amplitude decay of the radiated dilatational field components $\left(\dot{u}_{1}^{d}\right)$ and $\left(\dot{u}_{2}^{d}\right)$, plotted as a function of the normalized distance $\xi_{2} / \xi_{2}^{\max }$. The dilatational field components decay as $1 /\left(\xi_{2} / \xi_{2}^{\max }\right)^{q}$ in accordance with Eq. (12). Note that the amplitude decay will be generally slower than the $1 / \sqrt{r}$ decay observed by the sub-Rayleigh velocity field components, since $0 \leq q \leq 1 / 2$. 
(a)

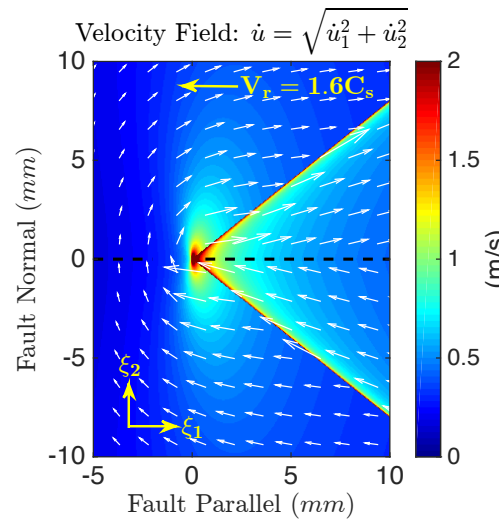

(c)

Fault Normal Particle Velocity Waveforms

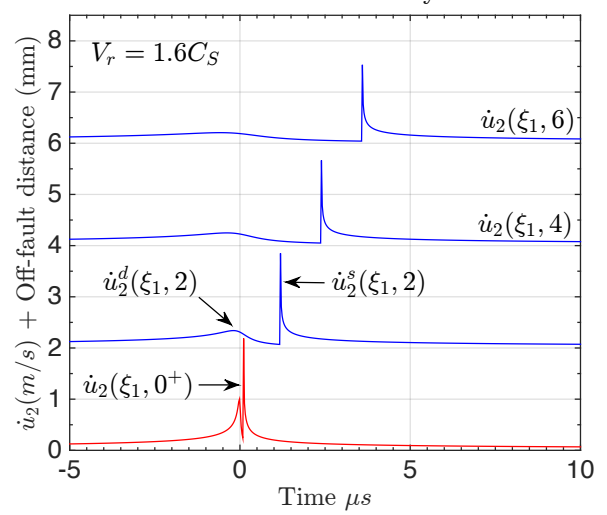

(b) Supershear Dilatational Field Decay

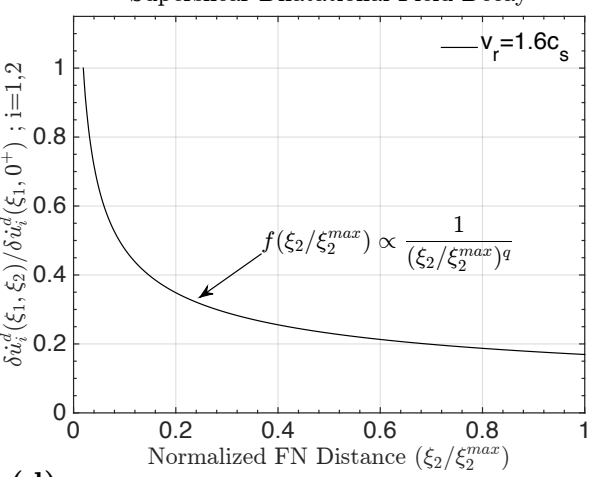

(d)

Fault Parallel Particle Velocity Waveforms

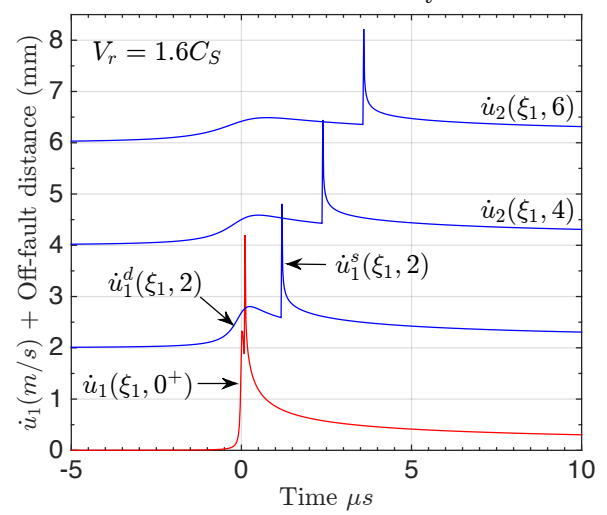

Figure 2: (a) Supershear velocity field plot for a right-lateral/left-traveling shear crack. (b) Normalized amplitude decay of the dilatational velocity field components. (c) Synthetic FN velocity waveforms at incremented distances from the fault. (d) Synthetic FP velocity waveforms at incremented distances from the fault. Note: The crack tip singularity was excluded in Figures (c,d) by normalizing with respect to the particle velocity $\dot{u}_{i}\left(\xi_{1}, 0^{+}\right)$, where $\xi_{2}=0^{+}$ is equivalent to $\xi_{2}=0.2 \mathrm{~mm}$ within the arbitrarily imposed length scale.

Figures 2(c,d) represent synthetic supershear particle velocity waveforms corresponding to the fault-parallel $\left(\dot{u}_{1}\right)$ and fault-normal $\left(\dot{u}_{2}\right)$ motion components. The near-fault velocity waveforms $\dot{u}_{i}\left(\xi_{1}, 0^{+}\right)$exhibit a pronounced particle velocity jump coinciding with the arrival of the rupture front, which results from the superposition of the dilatational and shear field contributions. The shear and dilatational fields propagate at their respective, characteristic elastic wave speeds and proceed to separate with increased distance and time as depicted in the off-fault waveforms. Each off-fault waveform is distinctly characterized by a pronounced velocity swing, attributed to the leading dilatational field lobe, which arrives in advance of the shear Mach front. The fault-parallel component of the dilatational field lobe exhibits a positive velocity swing $\left(\delta \dot{u}_{1}^{d}\right)$ that dominates in magnitude over the velocity swing $\left(\delta \dot{u}_{2}^{d}\right)$ of the fault-normal component.

The sharp, step-like velocity jumps in the off-fault synthetic waveforms co- 
incide with the shear Mach front. The ratio between the FP and FN velocity jumps at any point along the shear Mach front remains constant and is given

$$
\frac{\delta \dot{u}_{1}^{s}}{\delta \dot{u}_{2}^{s}}=\beta_{s} \operatorname{sgn}\left(\xi_{2}\right) ; \quad \text { (right-lateral/left-traveling). }
$$

Examination of the factor $\beta_{s}$ (Eq. 10 , reveals that $\delta \dot{u}_{1}^{s} / \delta \dot{u}_{2}^{s} \geq 1$ when $\sqrt{2} C_{s} \leq$ $V_{r} \leq C_{d}$. The motion propagated by shear Mach front is thus generally characterized by a pronounced fault-parallel velocity jump, which dominates in magnitude over the corresponding fault-normal velocity jump.

Dividing Eq. (8) by Eq. (9) yields a similar expression for a right-lateral/righttraveling supershear rupture, in which case, the ratio between the fault-parallel and fault-normal velocity jumps along the shear Mach fronts is given by

$$
\frac{\delta \dot{u}_{1}^{s}}{\delta \dot{u}_{2}^{s}}=-\beta_{s} \operatorname{sgn}\left(\xi_{2}\right) ; \quad \text { (right-lateral/right-traveling). }
$$

The 2D steady state solution predicts that the shear field velocity jumps $\left(\dot{u}_{i}^{s}\right)$ along the shear Mach front are sustained out to infinity. This property is demon405 strated by the sharp velocity jumps of equal magnitude in the stacked off-fault velocity waveforms. Any observed differences in the magnitudes of the velocity jumps in Figures 2(c,d) are a plotting artifact related to numerical sampling of the grid points used to generate the field plot. The constant amplitude of the shear field velocity components is in stark contrast to the pronounced broaden410 ing and rapid amplitude decay exhibited by the dilatational field components $\left(\dot{u}_{i}^{d}\right)$.

In a later section of this paper, a strong correspondence is demonstrated between the particle velocity records obtained from supershear LEQ experiments and the general profile and predicted sense of motion of the synthetic waveforms depicted in Figures 2(c,d). The rapid spatiotemporal separation between the dilatational and shear fields is also experimentally observed and exploited to probe the velocity field components $\dot{u}_{1}^{s}$ and $\dot{u}_{2}^{s}$ propagated by the shear Mach front.

\subsection{Predicted Sense of Particle Motion for Right-Lateral and Left-Lateral Su- pershear Rupture Velocity Fields}

The superposition of shear wavelets emitted by a steady-state, intersonic rupture front leads to the formation of a shear Mach cone, which stems from the advancing rupture tip and envelopes the surrounding medium along either side of the fault. The resulting Mach cone half-angle, measured with respect to the fault plane, is given by the familiar relation

$$
\theta=\sin ^{-1}\left(C_{s} / V_{r}\right)
$$

Inspection of Eq. 16 reveals that the Mach angle domain $\sin ^{-1}\left(C_{s} / C_{d}\right) \leq$ $\theta \leq \pi / 4$ corresponds to the maximum stable (supershear) rupture speed regime $\sqrt{2} C_{s} \leq V_{r} \leq C_{d}$, while $\pi / 4<\theta \leq \pi / 2$ corresponds to the unstable rupture 


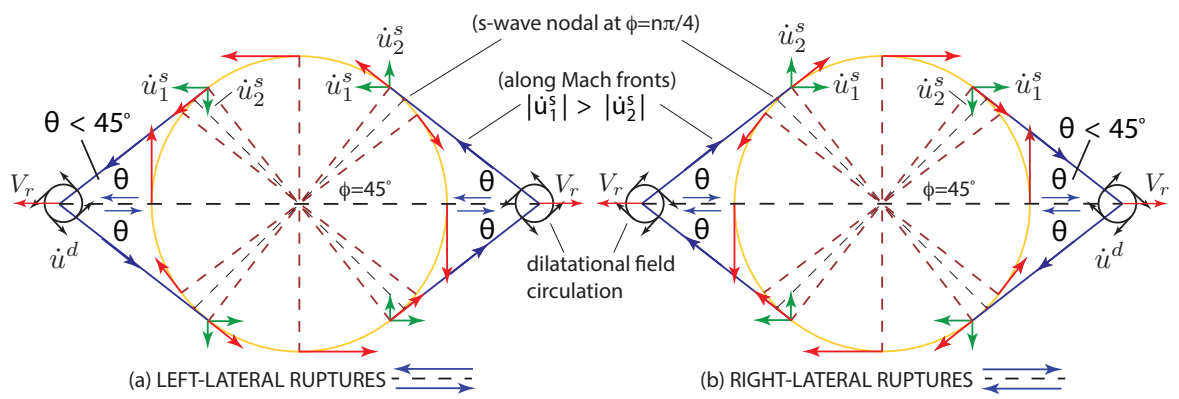

Sense of particle Motion in the stable supershear rupture speed regime

Figure 3: (a) Sense of particle motion for (stable) left-lateral, right and left traveling, supershear rupture velocity fields. (b) Sense of particle motion for (stable) right-lateral, right and left traveling, supershear rupture velocity fields.

speed domain $C_{s} \leq V_{r}<\sqrt{2} C_{s}$. Special cases of interest that set a lower bound for $\theta_{\text {min }}=\sin ^{-1}\left(C_{s} / C_{d}\right)$ (as $V_{r} \rightarrow C_{d}$ ) are (1) $C_{d}=\sqrt{3} C_{s}$, often a good approximation for crustal rock, in which case supershear Mach angles are restricted to $\sin ^{-1}(1 / \sqrt{3}) \leq \theta \leq \pi / 4$, and (2) $C_{d} \approx 2 C_{s}$, applicable to Homalite100 laboratory earthquake test specimens, in which case the supershear Mach angles are restricted to $\pi / 6 \leq \theta \leq \pi / 4$.

The diagrams in Figure 3 enable quick determination of the expected sense of particle motion of the dilatational field lobe and shear Mach fronts of a leftbilateral or right-bilateral supershear rupture propagating in the stable rupture speed regime $\sqrt{2} C_{s} \leq V_{r} \leq C_{d}$. The velocity fields predicted by Eqns. (8) and (9) describe a right-lateral/right-traveling rupture and thus correspond to the 440 right-half of the diagram in Figure 3 (b).

The large circle in each diagram represents a hypothetical S wavelet emitted by the rupture tip at an earlier time. The dashed black lines oriented at $\phi=$ $\pm 45^{\circ}$ with respect to the fault plane intersect the $\mathrm{S}$ wavefronts at the nodal points where $\dot{\vec{u}}^{s}=0$ and the sense of particle motion reverses. The shear Mach

${ }_{445}$ fronts arise from the constructive superposition of a continuum of $\mathrm{S}$ wavefronts emitted by the propagating rupture at earlier times. The blue lines stemming from the rupture tip and oriented at an angle $\theta$ with respect to the fault represent the shear Mach fronts. As previously noted, $\sin ^{-1}\left(C_{s} / C_{d}\right) \leq \theta \leq \pi / 4$ in the case of a stable supershear rupture. A shear Mach front must therefore lie tangent to the expanding $\mathrm{S}$ wavefront at a point located within the angular domain $\pi / 4 \geq \phi \leq \pi / 2-\sin ^{-1}\left(C_{s} / C_{d}\right)$, as depicted in Figure 3. The point of tangency is simply dictated by the rupture speed $V_{r}$ in accordance with Eq. 16 . The red arrows lying tangent to the circular $\mathrm{S}$ wavefront indicate the sense of particle motion at the point of tangency in accordance with the far-field $\mathrm{S}$ wave 455 radiation pattern produced by a shear dislocation Aki and Richards (2002). The magnitude and sense of particle motion along a shear Mach front is decomposed into fault-parallel and fault-normal particle velocity components $\left(\dot{u}_{1}^{s}\right.$ and $\left.\dot{u}_{2}^{s}\right)$, which are represented by the green arrows in Figure 3. 


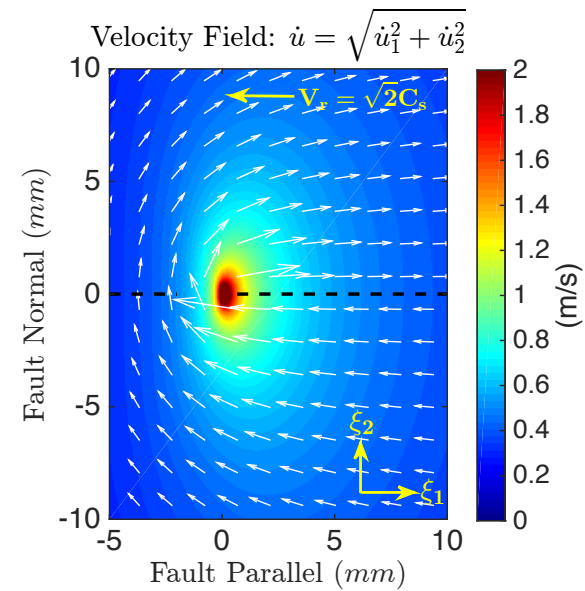

Figure 4: Particle velocity field of a right-lateral/left traveling supershear crack propagating at $V_{r}=\sqrt{2} C_{s}$. Shear Mach fronts vanish leaving only the dilatational velocity field lobe, characterized by a field amplitude, which scales as $1 / \sqrt{r}$, at this rupture speed.

The smaller black circles with tangent vectors, centered around the hypothetical rupture tips in each diagram, represent the dilatational velocity field $\left(\dot{u}^{d}\right)$, which circulates around the rupture tip in accordance with the kinematic condition $\nabla u^{d}=0$. Careful examination of the sense of motion corresponding to the right-lateral/left-traveling rupture in Figure 3(b) shows it to be in full agreement with the the velocity field plot and synthetic waveforms depicted in Figure 2.

The signs of the velocity components given by Eqns. (8) and (9) for a (rl/lt) supershear rupture may be readily adjusted in order to describe all other rupture scenarios portrayed in Figure 3, i.e., (1) the left-lateral/right-traveling rupture corresponding to the right-half of the diagram in Figure 3(a), (2) the left470 lateral/left-traveling rupture corresponding to the left-half of Figure 3(a), and (3) the right-lateral/left-traveling rupture corresponding to the left-half of the diagram in Figure 3(b). The latter case was previously considered to generate the plots depicted in Figure 2 .

\subsection{Vanishing of the $S$ wave field at $V_{r}=\sqrt{2} C_{s}$}

475 As the rupture speed $V_{r} \rightarrow \sqrt{2} C_{s}$, the Mach angle approaches $\theta=\pi / 4$ in accordance with Eq. (16). The shear Mach front must, therefore, vanish at this rupture speed since it is formed along a locus of nodal points positioned at $\phi=\pi / 4$ on the radiated $\mathrm{S}$ waves. This feature is a fundamental wavefront superposition property, which must occur as $V_{r} \rightarrow \sqrt{2} C_{s}$, irrespective of whatever dynamic rupture model is invoked. Indeed, the vanishing Mach front condition naturally emerges from the 2D steady state singular elastic solutions given by Eq. (7). Figure 4 depicts velocity field of a right-lateral/left-traveling supershear crack, propagating with rupture speed $V_{r}=\sqrt{2} C_{s}$. The dilatational field is now prominently displayed, with the shear Mach fronts conspicuously absent at this 
rupture speed. High speed images and particle velocity traces of a supershear rupture observed propagating at $V_{r} \approx \sqrt{2} C_{s}$ are presented and analyzed in a later section of this paper.

\section{Spatiotemporal Velocity Field Properties Derived from 2D Steady State (Non-singular) Cohesive Zone Models}

\subsection{Steady State Cohesive Zone Models}

The underlying assumption in the singular elastic crack models is that the rupture process is assumed to occur at a mathematically singular crack tip. A major weakness of this model is that it fails to account for a finite process zone and cohesive strength, which varies along the length of the rupture zone.

495 Non-singular, slip-weakening models for shear, or Mode II cracks, incorporate a description of the shear tractions within a finite breakdown zone Barenblatt (1962), Ida (1972), Palmer and Rice (1973). The shear traction is characterized by a peak strength just ahead of the crack tip, which decreases with increased distance behind the crack tip and then levels off to a residual shear strength level.

500 The incorporation of a cohesive zone in these models introduces a characteristic length scale into the analysis, which is distinctly missing in the singular elastic models. The resulting length scale is proportional to the size of the breakdown zone.

\subsection{D steady-state Intersonic Slip-Pulse with Prescribed Shear Traction within} a Finite Slip Zone

Dunham and Archuleta (2005) extended an earlier solution given by Broberg (1989), to obtain non-singular analytical solutions for the stress and particle velocity fields radiated by a $2 \mathrm{D}$ steady-state intersonic slip-pulse with an arbitrarily prescribed shear traction within a finite slip zone. The solution to the mixed boundary value problem is obtained by invoking linear superposition of the constant far field stresses $\sigma_{i j}^{f}\left(\xi_{1}, \xi_{2}\right)=\sigma_{11}^{0}+\sigma_{22}^{0}+\tau_{0}$, imposed upon the fault at all times, and the local stress field problem, which is solved, subject to the prescribed traction $\tau\left(\xi_{1}\right)-\tau_{0}$ across the slip zone.

The mixed boundary value problem leads to a non-singular solution for the particle velocity field components of a ( $\mathrm{rl} / \mathrm{rt})$ intersonic slip pulse are given by

$$
\begin{gathered}
\dot{u}_{1}=-\frac{V_{r}}{\mu}\left[\frac{1}{2 \alpha_{d}} \Im N\left(z_{d}\right)+\frac{\beta_{s}^{2}-1}{4 \alpha_{d}} \Im N\left(z_{s}\right)\right] \\
\dot{u}_{2}=-\frac{V_{r}}{\mu}\left[\frac{1}{2} \Re N\left(z_{d}\right)-\operatorname{sgn}\left(\xi_{2}\right) \frac{\beta_{s}^{2}-1}{4 \alpha_{d} \beta_{s}} \Im N\left(z_{s}\right)\right]
\end{gathered}
$$

where the variables $z_{d}, z_{s}$ are given by

$$
z_{d}=\xi_{1}+i \alpha_{d} \xi_{2} \quad ; \quad z_{s}=\xi_{1} \pm \beta_{s} \xi_{2}
$$




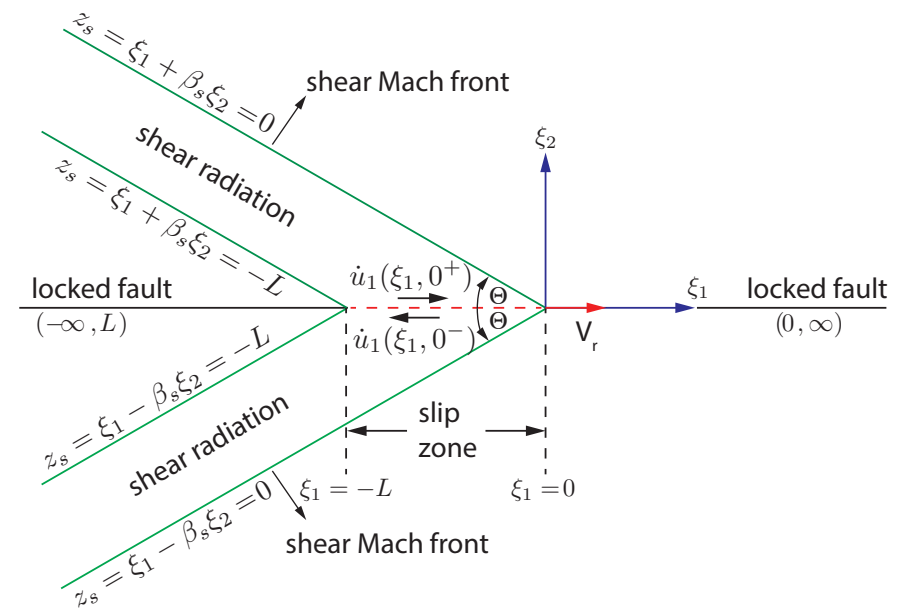

Figure 5: $\mathrm{S}$ wave radiation field bounded by shear Mach fronts extending from the leading and trailing edges of the supershear slip zone

Solutions for the stress and particle velocity fields are expressed in terms of a single integral expression for $\tau\left(\xi_{1}\right)$ over the slip zone, given by

$$
N(z)=-\frac{\sin (\pi q)}{\pi} z^{1-q}(z+L)^{q} \int_{-L}^{0} \frac{\tau(w)-\tau_{0}}{(-w)^{1-q}(w+L)^{q}(w-z)} d w
$$

520

which corresponds to a complex integral solution of a Hilbert problem. The function $N(z)$ provides the link between the shear traction $\tau\left(\xi_{1}\right)$ within the slip zone and the explicit form of the stress and particle velocity fields radiated by the $2 \mathrm{D}$ steady state intersonic slip pulse. In the case of a sliding mode II rupture, $\tau\left(\xi_{1}\right)$ represents the frictional resistance (cohesion), which stems from a governing friction law. $N(z)$ is analytic everywhere, except along the branch cut $\left(-L<\xi_{1}<0\right)$ corresponding to the slip zone. Careful examination of Eq. (20) at points very close to the fault (letting $z_{I} \rightarrow 0$ ) reveals that $N(z)$ is a complex function only within the interval $\left(-L<\xi_{1}<0\right)$ (Dunham and Archuleta,

530 2005). This implies that $\mathrm{S}$ wave radiation is confined within a region of space bounded by pairs of leading and trailing characteristics $z_{s}=\xi_{1} \pm \beta_{s} \xi_{2}=c$, $(c=$ constant $)$, corresponding to the shear Mach fronts, which extend from the leading and trailing edges of the slip zone, as depicted in Figure 5. In the case of a right-lateral/right-traveling slip pulse, the characteristic defined by ${ }_{535} z_{s}=\xi_{1}+\beta_{s} \xi_{2}=0$ describes the leading, negatively-sloped shear Mach front within the upper half-space $\left(\xi_{2}>0\right)$, while the characteristic $z_{s}=\xi_{1}-\beta_{s} \xi_{2}=0$ corresponds to the leading positively-sloped shear Mach front within the lower half-space $\left(\xi_{2}<0\right)$.

The "sign function" $\operatorname{sgn}\left(\xi_{2}\right)$ in Eq. 18 accounts for the anti-symmetry of $\Im N\left(z_{s}\right)$ with respect to the fault plane and extends the solution into the lower half-space $\left(\xi_{2}<0\right)$. The fault-normal velocity component would otherwise 
abruptly reverse sign across the fault, which is prohibited in the case of a mode II rupture. Numerical solutions for Eq. (17) and Eq. (18) were obtained by Dunham and Archuleta (2005) using a linear distance-weakening model for $\tau\left(\xi_{1}\right)$, originally formulated by Palmer and Rice (1973). Due to the finite crack-tip stresses and finite width of the process zone, the resulting particle velocity waveforms are of finite width and magnitude, unlike the waveforms, which emerge from the singular elastic solution. Indeed, the singular elastic solution given by Freund (1979) emerges as a special case of the 2D steady state slip pulse model 550 in the limit $(R / L \rightarrow 0)$. Also, in stark contrast to the singular models, the crack-tip energy flux is non-zero and finite in the supershear regime (Broberg, 1989: Samudrala et al., 2002b a; Rosakis et al., 2007).

The dilatational and shear field contributions are each uniquely represented by separate terms in the solutions given by Eqs. (17) and (18). The first term in each equation corresponds to the dilatational field contribution, expressed in terms of the real and imaginary parts of $N\left(z_{d}\right)$ and is evaluated at points $z_{d}=\xi_{1}+i \alpha_{d} \xi_{2}$ in the complex plane, i.e., $\Im N\left(z_{d}\right)$ in Eq. (17) and $\Re N\left(z_{d}\right)$ in Eq. (18). The shear field terms are both functions of $\Im N\left(z_{s}\right)$, and are evaluated exclusively along the family of characteristics defined by $z_{s}=\xi_{1} \pm \beta_{s} \xi_{2}=c$.

We may also consider the limiting case, where the dilatational field contribution has decayed away, leaving only the unattenuated fault-parallel and fault-normal shear field velocity components, which are propagated into the far field along the shear Mach fronts. Dividing the shear field term of Eq. (17) by the corresponding shear field term of Eq. (18) recovers the previously noted scaling relationship given by Eq. (15) between the FP and FN velocity components propagated by the shear Mach front of a $(\mathrm{rl} / \mathrm{rt})$ rupture. The relationship is now expressed in terms of the complex (characteristic) variable $z_{s}=\xi_{1} \pm \beta_{s} \xi_{2}$, and given by

$$
\frac{\delta \dot{u}_{1}\left(z_{s}\right)}{\delta \dot{u}_{2}\left(z_{s}\right)}=-\operatorname{sgn}\left(\xi_{2}\right) \beta_{s} ; \quad \text { (right-lateral/right-traveling). }
$$

In the case of a (rl/lt) supershear rupture, such as those observed in our experi570 ments, the ratio between the fault-parallel and fault-normal motion components propagated by the shear Mach fronts is given by

$$
\frac{\delta \dot{u}_{1}\left(z_{s}\right)}{\delta \dot{u}_{2}\left(z_{s}\right)}=\operatorname{sgn}\left(\xi_{2}\right) \beta_{s} ; \quad \text { (right-lateral/left-traveling), }
$$

which recovers Eq. 14.

5.3. Scaling Relationship Between the Frictional Fault Sliding Speed and the Fault-Parallel Components of the Dilatational Field and Shear Mach Front

The slip function along the fault $\left(\xi_{2}=0\right)$ is defined as

$$
\Delta \dot{u}_{1}\left(\xi_{1}, 0\right)=\dot{u}_{1}\left(\xi_{1}, 0^{+}\right)-\dot{u}_{1}\left(\xi_{1}, 0^{-}\right)
$$


where $\dot{u}_{1}\left(\xi_{1}, 0^{+}\right)$and $\dot{u}_{1}\left(\xi_{1}, 0^{-}\right)$represent the fault-parallel velocity components at two infinitesimally separated points positioned just above and below the fault. We note that since $z_{d}=z_{s}=\xi_{1}$ as $x_{2} \rightarrow 0^{+}$, Eqn. (17) then assumes the form

$$
\dot{u}_{1}\left(\xi_{1}, 0^{+}\right)=-\frac{V_{r}}{\mu}\left[\frac{1}{2 \alpha_{d}} \Im N^{+}\left(\xi_{1}\right)+\frac{\beta_{s}^{2}-1}{4 \alpha_{d}} \Im N^{+}\left(\xi_{1}\right)\right]
$$

where

$$
\begin{gathered}
N^{+}\left(\xi_{1}\right)=\lim _{\xi_{2} \rightarrow 0^{+}} N(z)=\frac{\sin (\pi q)}{\pi} e^{-i \pi q}\left[\left(-\xi_{1}\right)^{1-q}\left(\xi_{1}+L\right)^{q} \times\right. \\
\left.\int_{-L}^{0} \frac{\tau(w)-\tau_{0}}{(-w)^{1-q}(w+L)^{q}\left(w-\xi_{1}\right)} d w+i \pi\left[\tau\left(\xi_{1}\right)-\tau_{0}\right]\right]
\end{gathered}
$$

580 is a complex expression containing a real integral, which is evaluated within the slip zone $\left(-L<\xi_{1}<0\right)$. We note the presence of the familiar rupture speed dependent factor $q$ given by Eq. (11), which continues to govern the rate at which the field strength decays with increased distance from the fault plane. Invoking mode II anti-symmetry of the fault-parallel component, i.e., $\Delta \dot{u}_{1}\left(\xi_{1}, 0\right)=2 \dot{u}_{1}\left(\xi_{1}, 0^{+}\right)$, in combination with Eq. (24), leads to an alternate expression for the slip function given by

$$
\Delta \dot{u}_{1}\left(\xi_{1}, 0\right)=-2 \frac{V_{r}}{\mu}\left(\frac{\beta_{s}^{2}+1}{4 \alpha_{d}}\right) \Im N^{+}\left(\xi_{1}\right) .
$$

Similarly, since $\Delta \dot{u}_{1}\left(\xi_{1}, 0\right)=-2 \dot{u}_{1}\left(\xi_{1}, 0^{-}\right)$and since $\Im N^{+}\left(\xi_{1}\right)=-\Im N^{-}\left(\xi_{1}\right)$ (mode II antisymmetry), it follows that

$$
\Delta \dot{u}_{1}\left(\xi_{1}, 0\right)=2 \frac{V_{r}}{\mu}\left(\frac{\beta_{s}^{2}+1}{4 \alpha_{d}}\right) \Im N^{-}\left(\xi_{1}\right) .
$$

and thus,

$$
\Delta \dot{u}_{1}\left(\xi_{1}, 0\right)=\mp 2 \frac{V_{r}}{\mu}\left(\frac{\beta_{s}^{2}+1}{4 \alpha_{d}}\right) \Im N^{ \pm}\left(\xi_{1}\right) .
$$

The somewhat ambiguous form given by Eq. 28) is cast into a more compact expression by invoking $\Delta \dot{u}_{1}\left(\xi_{1}, 0\right)=\Delta \dot{u}_{1}\left(z_{s}\right)$ and $\Im N^{+}\left(\xi_{1}\right)=\Im N\left(z_{s}\right)$, where $z_{s}=\xi_{1} \pm \beta_{s} \xi_{2}=c$ defines the characteristics (shear Mach fronts), which extend to infinity within each respective half-space. We may then write

$$
\Delta \dot{u}_{1}\left(z_{s}\right)=-\operatorname{sgn}\left(\xi_{2}\right) \frac{2 V_{r}}{\mu}\left(\frac{\beta_{s}^{2}+1}{4 \alpha_{d}}\right) \Im N\left(z_{s}\right)
$$

where the function $\operatorname{sgn}\left(\xi_{2}\right)$ is introduced in order to preserve the sign of $\Delta \dot{u}_{1}\left(z_{s}\right)$ regardless of which characteristic is assumed.

Experimental estimates of the frictional fault sliding speed are obtained in our supershear experiments by focusing a laser vibrometer beam at nearly grazing incidence onto a point positioned within $200 \mu \mathrm{m}$ above frictional fault plane. 
The near-fault sliding speed is directly compared to the FP velocity component propagated by the shear Mach front, measured at an off-fault field point as the Mach front sweeps past this location. Details of the vibrometer probe configurations are provided in the experimental methods section. The experiments as mentioned above provide the motivation to recast Eq. (17) into an alternative "near-fault form", which will prove very useful in the analysis and interpreta605 tion of the on-fault and off-fault particle velocity waveforms obtained in these experiments.

Eq. (24) indicates that a temporally resolved "on-fault" record of the frictional fault sliding speed $\dot{u}_{1}\left(\xi_{1}, 0^{+}, t\right)$ is equivalent to measuring $\Im N^{+}\left(\xi_{1}, t\right)$. Substituting for $\Im N^{+}\left(\xi_{1}\right)$ in Eq. 24), using the slip function relation given by Eq. (26), and invoking anti-symmetric slip function relation $\dot{u}_{1}\left(\xi_{1}, 0^{+}\right)=$ $\frac{1}{2} \Delta \dot{u}_{1}\left(\xi_{1}, 0\right)$ leads to

$$
\dot{u}_{1}\left(\xi_{1}, 0^{+}\right)=\left(\frac{2}{\beta_{s}^{2}+1}\right) \dot{u}_{1}\left(\xi_{1}, 0^{+}\right)+\left(\frac{\beta_{s}^{2}-1}{\beta_{s}^{2}+1}\right) \dot{u}_{1}\left(\xi_{1}, 0^{+}\right)
$$

We note that the first and second terms to the right of the equality symbol must still correspond to the dilatational and shear field contributions, respectively. Equation (30) thus reveals how the frictional fault sliding speed $\dot{u}_{1}\left(\xi_{1}, 0^{+}\right)$, is partitioned between the dilatational and shear field radiation terms in accordance with the rupture speed dependent amplitude scaling factors appearing in parentheses. Hence we may define,

$$
r_{d}=\frac{\dot{u}_{1}^{d}\left(\xi_{1}, 0^{+}\right)}{\dot{u}_{1}\left(\xi_{1}, 0^{+}\right)}=\frac{2}{\beta_{s}^{2}+1}
$$

as the fault-parallel amplitude scaling factor that dictates how much of the frictional fault sliding speed $\dot{u}_{1}\left(\xi_{1}, 0^{+}\right)$is converted into the fault-parallel component of the dilatational field and subsequently radiated away.

A second fault-parallel amplitude scaling factor given by

$$
r_{s}=\frac{\dot{u}_{1}^{s}\left(\xi_{1}, 0^{+}\right)}{\dot{u}_{1}\left(\xi_{1}, 0^{+}\right)}=\frac{\beta_{s}^{2}-1}{\beta_{s}^{2}+1}
$$

is similarly defined, which governs how much of the frictional fault sliding speed $\dot{u}_{1}\left(\xi_{1}, 0^{+}\right)$is converted into the fault-parallel component of the shear field $\dot{u}_{1}^{s}\left(z_{s}\right)$ and subsequently propagated out into the far field along a shear Mach front.

Equations (31) and 32 represent a non-singular, 2D steady-state, scaling relationship between the sliding component induced by the supershear rupture at the fault plane and the fault-parallel component propagated by the shear Mach front. A similar relation naturally applies on the opposite side of the fault plane, between the frictional fault sliding speed $\dot{u}_{1}^{d}\left(\xi_{1}, 0^{-}\right)$, and the shear ${ }_{630}$ field velocity component $\dot{u}_{1}^{s}\left(z_{s}\right)$, where $z_{s}=\xi_{1}-\beta_{s} \xi_{2}$ corresponds to the shear Mach front within the lower half-space $\xi_{2}<0$.

Alternative and simplified forms for $r_{s}$ and $r_{d}$, given by

$$
r_{d}=2 \frac{C_{s}^{2}}{V_{r}^{2}} \quad ; \quad r_{s}=1-2 \frac{C_{s}^{2}}{V_{r}^{2}}
$$




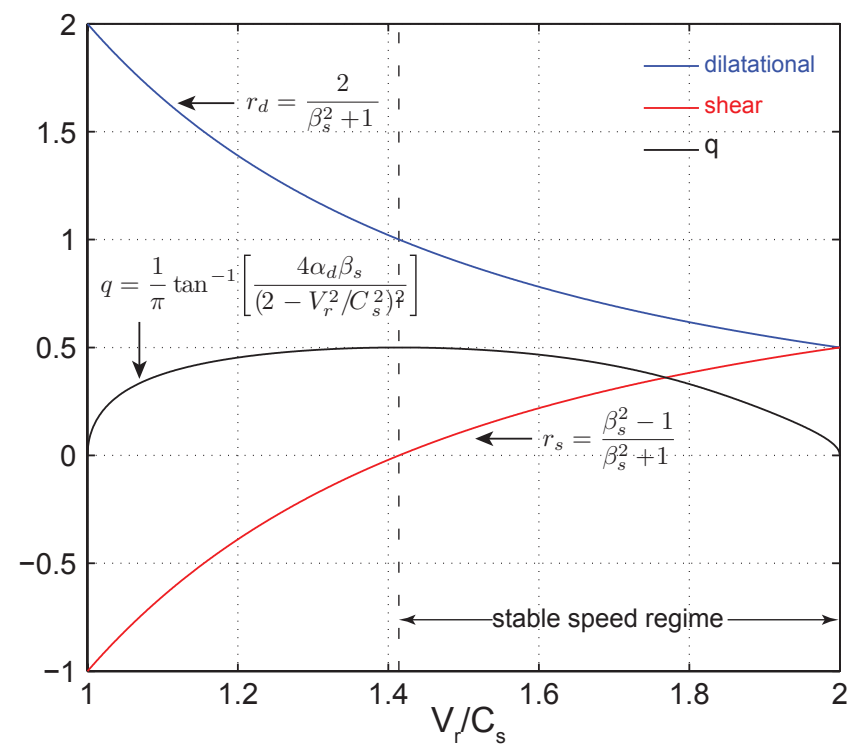

Figure 6: Non-singular scaling relationships between the frictional fault sliding speed $\dot{u}_{1}\left(\xi_{1}, 0^{+}\right)$and the fault-parallel velocity components of the dilatational field $\dot{u}_{1}^{d}\left(\xi_{1}, \xi_{2}\right)$ and shear Mach front $\dot{u}_{1}^{s}\left(\xi_{1}, \xi_{2}\right)$.

are obtained by substituting for $\beta_{s}$ through Eqn. 10 , where $r_{s}+r_{p}=1$ is naturally observed.

Figure 6 depicts the graphical relationship between the amplitude scaling factors $r_{d}, r_{s}$ given by Eq. (31) and Eq. (32) and the normalized rupture speed $V_{r} / C_{s}$. A plot of the rupture speed dependent factor $q$, given by Eq. (11) is also included in the figure. The vertical dashed line at $V_{r} / C_{s}=\sqrt{2}$ denotes the boundary between the unstable and stable rupture speed domains.

The maximum normalized rupture speed $V_{r} / C_{s}=2$ in Figure 6 corresponds to the limit $V_{r} \rightarrow C_{d}$, for a material with $C_{d}=2 C_{s}$ such as the Homalite-100 material used in LEQ experiments. The dilatational scaling factor is bounded between $0.5 \leq r_{d} \leq 1$ within the stable rupture speed regime, reaching a max645 imum value $r_{d}=1$ when $V_{r} / C_{s}=\sqrt{2}$ and decreasing with increased rupture speed toward a minimum value of $r_{d}=0.5$ as $V_{r} \rightarrow C_{d}$. The shear field scaling factor $\left(r_{s}=1-r_{d}\right)$ is bounded between $0 \leq r_{s} \leq 0.5$ within the stable rupture speed regime, and ranges from a minimum value $r_{s}=0$ when $V_{r} / C_{s}=\sqrt{2}$ to a maximum value $r_{s}=0.5$ as $V_{r} \rightarrow C_{d}$.

The fact that $\dot{u}_{1}^{s}=0$ as $V_{r} / C_{s} \rightarrow \sqrt{2}$, implies that $\dot{u}_{2}^{s}=0$ in light of Eq. 21) or Eq. (22). Thus, there are no $\mathrm{S}$ waves radiated at this unique rupture speed, which is consistent with the heuristic $\mathrm{S}$ wave nodal point argument given earlier that the shear Mach front must vanish as $V_{r} / C_{s} \rightarrow \sqrt{2}$. We also note that $q=1 / 2$ at $V_{r} / C_{s} \rightarrow \sqrt{2}$ thus revealing that the dilatational field will decay as ${ }_{655} 1 / \sqrt{r}$, in accordance with Eq. 12 . Energy, which would otherwise be radiated by shear waves, is instead consumed by fracture at this rupture speed. 
The portion of the curves corresponding to the unstable rupture speed regime $\left(C_{s} \leq V_{r}<\sqrt{2} C_{s}\right)$ appears somewhat odd and confusing at first glance, since $-1 \leq r_{s}<0$ and $1<r_{d} \leq 2$ within this domain. The situation is best understood by referring back to Figure 3 and noting that the shear Mach front will be tangent to the $\mathrm{S}$ wavelets along points corresponding to $(0<\phi \leq \pi / 4)$ when $C_{s} \leq V_{r}<\sqrt{2} C_{s}$. The fault-normal component is now directed upward and dominates in magnitude over the fault-parallel component, which is directed opposite to the assumed direction of slip. Thus $\dot{u}_{1}^{s}\left(z_{s}\right)<0$ even though the

${ }_{665}$ sliding speed $\dot{u}_{1}\left(\xi_{1}, 0^{+}\right)$is positive. The negative range of values exhibited by $r_{s}$ simply reflects the fact that $\dot{u}_{1}^{s}\left(z_{s}\right)<0$ when $C_{s} \leq V_{r}<\sqrt{2} C_{s}$. The corresponding positive range of values $1<r_{d} \leq 2$ for the dilatational field contribution $\dot{u}_{1}^{d}\left(z_{s}\right)$ offset the negative contribution of the shear field so as to satisfy $r_{s}+r_{d}=1$ within the unstable rupture speed domain.

Finally, we note that there is also no known counterpart of Eq. (30), which applies to sub-Rayleigh rupture field components, nor is there an analogous scaling relationship, which applies to the fault-normal component $\dot{u}_{2}\left(\xi_{1}, 0^{+}\right)$of a supershear rupture. The presence of $\Re N\left(z_{d}\right)$ in the dilatational field term and $\Im N\left(z_{s}\right)$ in the shear field term of Eq. (18) (and no anti-symmetry relation for $675 \dot{u}_{2}$ ) prohibits the types of substitutions and algebraic manipulations that were adopted in the case of the fault-parallel component $\left(\dot{u}_{1}\right)$.

Thus, Eqs. (31) and (32) represent unique scaling relationships, which emerge from the 2D state slip pulse solution and strictly apply to the fault-parallel velocity (and normal stress $\sigma_{11}$ ) component of a supershear rupture field. The result is readily extended to the analysis and interpretation of crack-like velocity profiles such as those observed and recorded in LEQ experiments. In such cases, the crack-like rupture is viewed as a very broad slip pulse in the limiting sense $R / L \rightarrow 0$, with a finite rupture zone $R$ and a very large slip zone (i.e., $L \rightarrow \infty$ ). When viewed in this limiting sense one may then apply Eqs. (31) and (32) to the analysis of the leading edge of recorded fault-parallel and fault-normal velocity waveforms obtained in supershear experiments.

\section{Unsteady Cohesive Zone Models and the Spontaneous Nucleation of Supershear Ruptures}

Space and time are essentially equivalent in 2D steady-state rupture models. Such models implicitly assume that a rupture has existed for all time and thus cannot account for the rupture history and dynamic mechanisms that lead to the emergence of the supershear rupture in the first place. Various supershear rupture transition mechanisms have been proposed and examined using selfsimilar analytical models and numerical simulations. Such models typically rely upon an initial sub-Rayleigh rupture, which spontaneously nucleates and spawns an intersonic "daughter-crack" as a consequence of a critical dynamic stress condition. In the original Burridge-Andrews model (Andrews, 1976, Burridge, 1973 : Burridge et al., 1979), a peak in the S wave field positioned just ahead of the sub-Rayleigh rupture front steadily increases in magnitude until it overcomes

the frictional fault strength. Once this occurs a so-called "daughter crack" is 


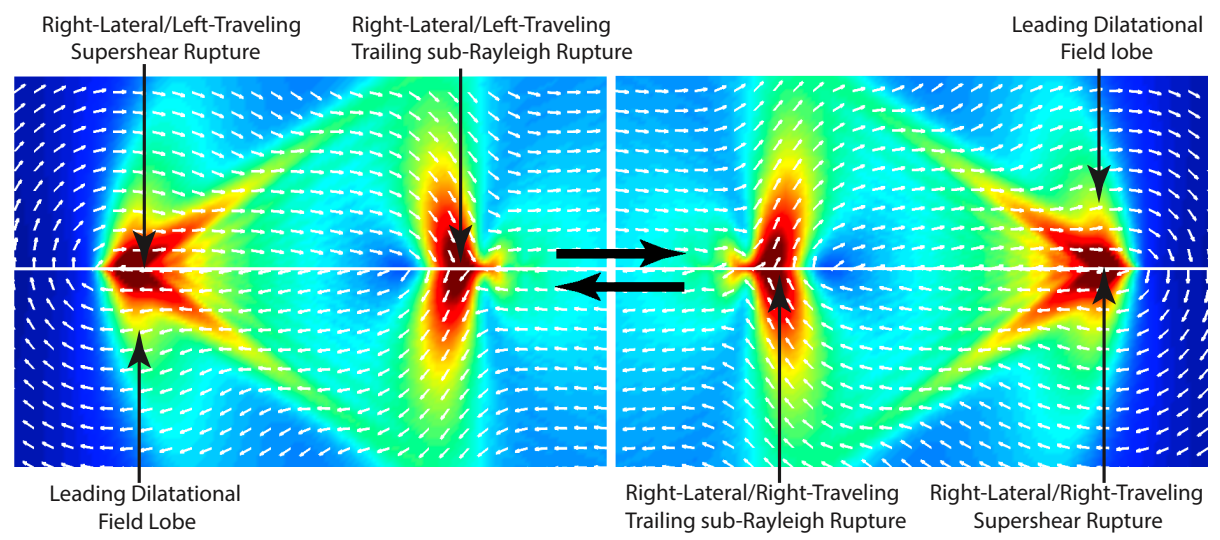

Figure 7: Contour and velocity vector field plots corresponding to right-lateral/left-traveling and right-lateral/left-traveling supershear ruptures. Field plots were generated by the finite element code ABAQUS, using a slip-weakening friction law in a numerical simulation of the Burridge-Andrews mechanism. Contour plot colors scale with the magnitude of the velocity field, while the streak plot vectors reveal the sense of particle motion throughout the field.

formed, which is momentarily dissociated from the original ruptured portion of the fault. The leading front of the daughter crack begins as an unstable intersonic rupture, which then rapidly accelerates and transitions into a stable supershear rupture.

Alternative and equally plausible models for the nucleation of supershear ruptures consider the dynamic interaction of the sub-Rayleigh rupture with an inclusion or a local patch of higher strength along the frictional fault. The presence of these features can also directly lead to dynamic stress concentrations and localized instabilities, which can give rise to a supershear rupture (Dunham 2003 Dunham and Archuleta 2004: Liu and Lapusta 2008). Each of the aforementioned supershear rupture transition mechanisms has been experimentally demonstrated and observed in LEQ experiments (Mello et al., 2010, 2014).

The graphical illustration in Figure 7 depicts the velocity fields and sense of particle motion in the immediate region surrounding a pair of left- and righttraveling (right-lateral) supershear ruptures. The analysis was conducted using the commercial Finite Element code, ABAQUS. The field plots were generated by assuming a slip-weakening friction law in a numerical simulation of the Burridge-Andrews mechanism (Andrews, 1976). Secondary sub-Rayleigh rup720 tures, shown trailing in the wake of the shear Mach cones are commonly observed in LEQ experiments. The left panel in Figure 7 corresponds to the velocity field of a right-lateral/left-traveling supershear rupture while the panel on the right represents a right-lateral/right-traveling supershear rupture velocity field. The colors of the contour plot scale with the magnitude of the velocity field, while the 725 streak plot vectors indicate the sense of particle motion throughout the field. Finally, we note how the particle motion of the numerically simulated rightlateral/left-traveling, trailing sub-Rayleigh and supershear ruptures in Figure 7 


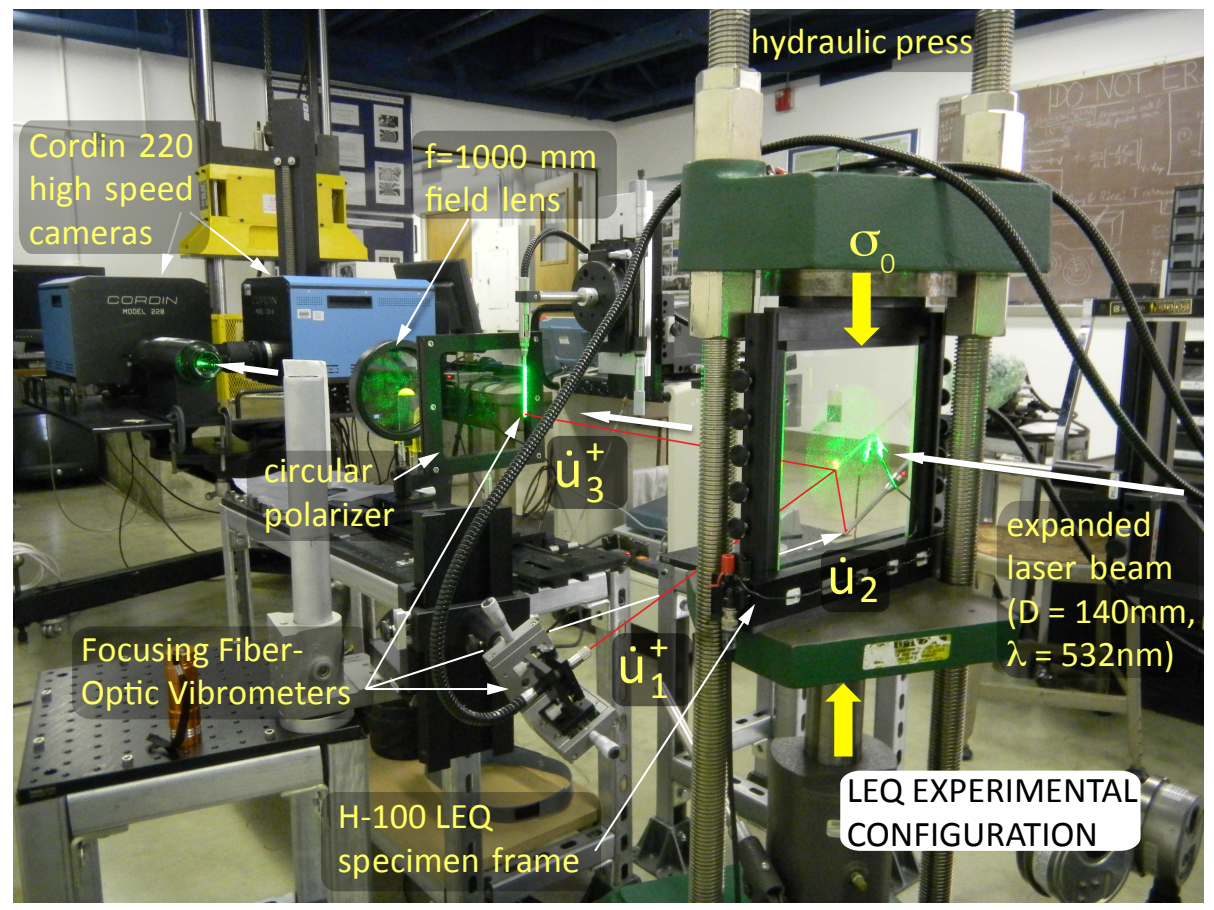

Figure 8: Laboratory earthquake experimental configuration featuring an integrated photoelastic interferometer and high-speed gated intensified cameras for full-field visualization of photoelastic interference patterns. Three fiber-optic laser vibrometers enable simultaneous monitoring of the in-plane $\left(\dot{u}_{1}, \dot{u}_{2}\right)$ and out-of-plane $\left(\dot{u}_{3}\right)$ surface velocity components at fixed field points on the surface of the Homalite-100 test specimen.

correlates with the predicted sense of particle motion of the sub-Rayleigh and supershear rupture fields depicted in Figures 12 .

\section{Materials and Methods}

\subsection{The Laboratory Earthquake Experiment (LEQ)}

The traditional LEQ experiment (Xia et al., 2004) features a transparent, $150 \mathrm{~mm} \times 150 \mathrm{~mm}$ square test specimen formed by two statically compressed, $10 \mathrm{~mm}$ thick, trapezoidal sections of Homalite-100, a linear elastic, and stress-

735 birefringent polymer. The mating fault surfaces are each initially polished to a flat, and nearly optical-grade surface finish to remove all machining-induced surface defects. The surfaces are then uniformly roughened to a nominal RMS surface roughness of $1.5 \mu \mathrm{m}$ using a prescribed and repeatable micro-bead blasting procedure. The inclined frictional interface formed by the mated surfaces of the statically compressed test sections mimics a tectonic fault plane within the Earth's crust. Other frictional fault configurations have also been developed and implemented in recent years, which emulate partially locked strike-slip faults 

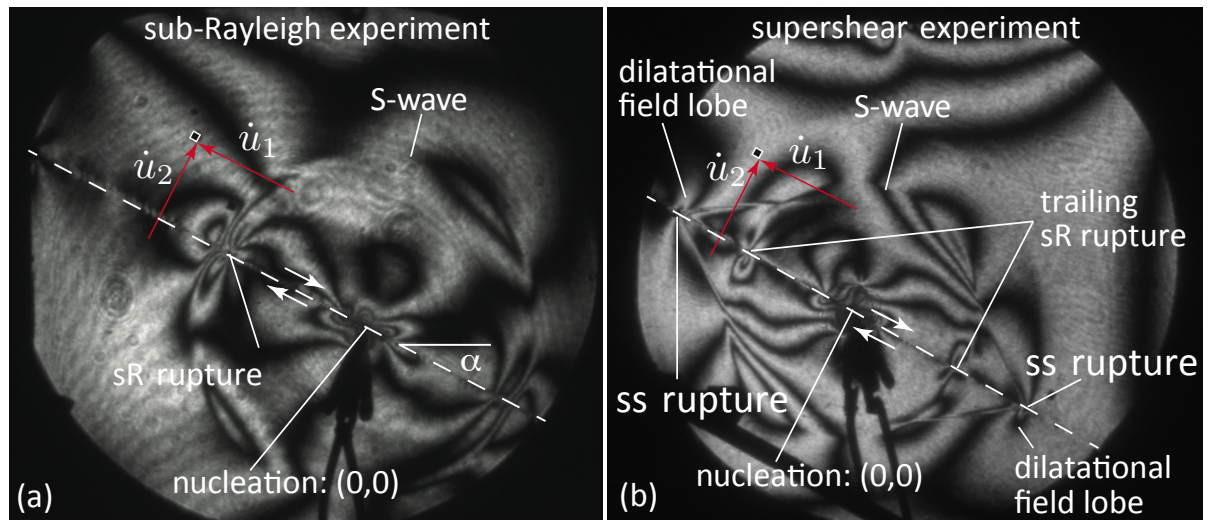

Figure 9: (a) High-speed photoelastic image of a right-bilateral sub-Rayleigh rupture and expanding $S$ wavefront. Red arrows depict how vibrometer beams are focused at an offault field point to monitor $\dot{u}_{1}$ and $\dot{u}_{2}$ as the rupture field sweeps past this location. (b) Right-bilateral supershear rupture characterized by prominent shear Mach fronts, with leading dilatational field lobes, and trailing sub-Rayleigh ruptures.

and normal thrust faults within the Earth's crust (Mello et al., 2014, Gabuchian et al. 2014).

The Homalite-100 LEQ test specimen is initially subjected to a static compressive load $\sigma_{0}$ using a mechanical, hydraulic press such as the one pictured in Figure 8. The compressive load translates into a normal traction $\sigma_{22}^{0}=\sigma_{0} \cos ^{2} \alpha$ and resolved shear traction $\sigma_{21}^{0}=\sigma_{0} \sin \alpha \cos \alpha$ along the frictional fault plane, where $\alpha$ represents the inclination angle of the fault plane with respect to the horizontal. Interfacial sliding is initially prevented by the fact that the resolved shear stress does not exceed the peak (static) frictional strength $\tau_{p}=f_{s} \sigma_{22}^{0}$ of the interface, where $f_{s}$ represents the static coefficient of friction. Rupture nucleation is triggered by the rapid ablation of a thin $\mathrm{NiCr}$ wire filament (diameter $\approx 80 \mu m$ ), which is fed through a small cylindrical cavity formed by

755 a pair of matching semicircular cylindrical grooves machined onto each of the mated fault surfaces (Mello et al. 2010).

A unique advantage of the LEQ experiment is the flexibility it offers to nucleate sub-Rayleigh or supershear ruptures depending upon the initial static compressive load $\left(\sigma_{0}\right)$, frictional fault angle $(\alpha)$, and other properties such 760 as the RMS surface roughness, and cohesion between the mated fault surfaces. Spatially-resolved photoelastic images recorded by high-speed cameras enable direct visualization and frame-by-frame tracking of critical features of sub-Rayleigh and supershear rupture fields. Figure 9 contrasts a sub-Rayleigh experiment and a supershear experiment. The photoelastic image in Figure 7659 (a) depicts a right bi-lateral sub-Rayleigh rupture, which was nucleated at the point labeled $(0,0)$. Figure 9(b) reveals a pair of supershear ruptures; a rightlateral/left traveling rupture and a right-lateral/right traveling rupture, each of which transitioned at an earlier time from the (labeled) trailing sub-Rayleigh ruptures. 

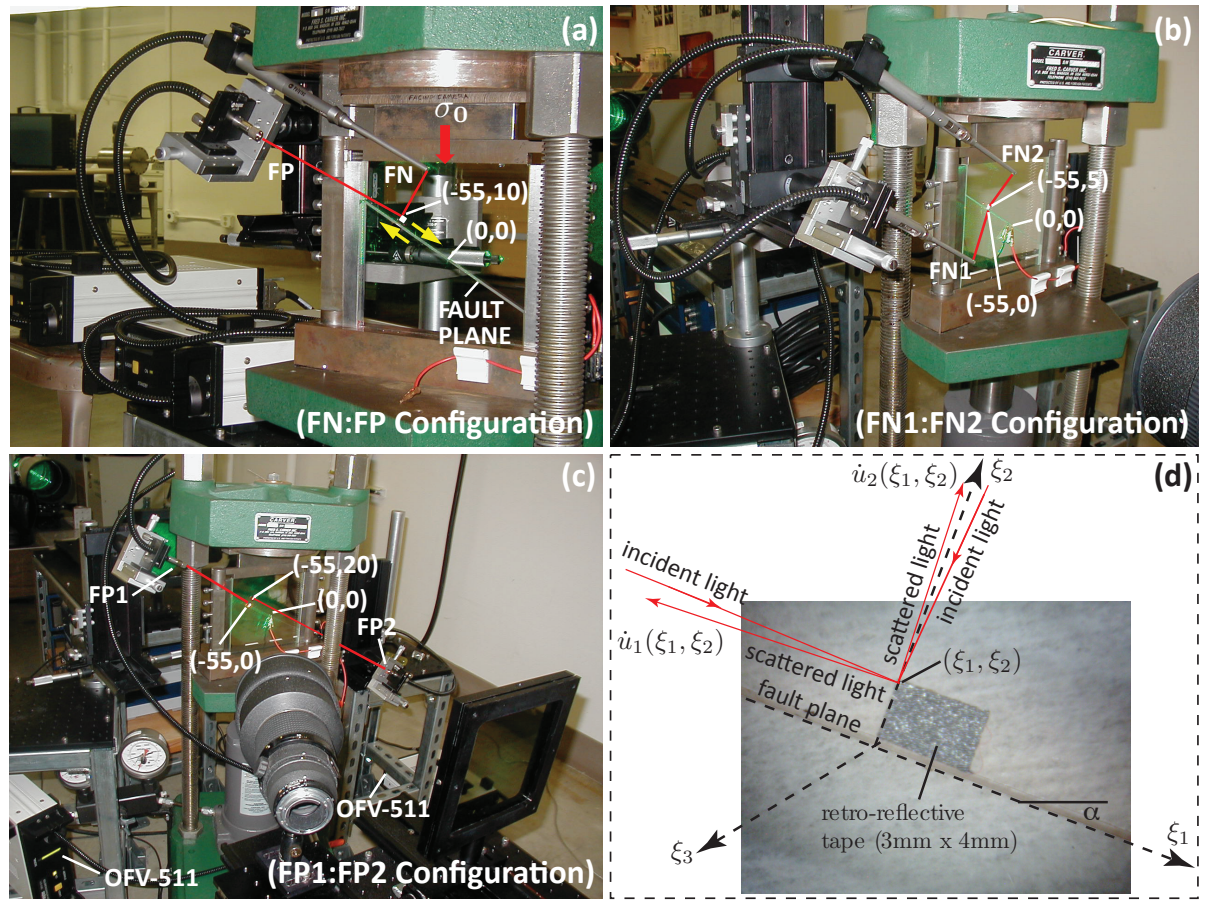

Figure 10: Laser vibrometer configurations: (a) (FN:FP) for simultaneous measurement of fault-normal and fault-parallel velocity components at a fixed field point. (b) (FN1:FN2) for simultaneous measurement of fault-normal velocity components at a pair of -on and off-fault field points. (c) (FP1:FP2) for simultaneous measurement of fault-parallel velocity components at a pair of -on and off-fault field points. (d) Use of retro-reflective tape corner for efficient scattering of focused laser beam.

\subsection{Laser Vibrometer Configurations Applied to Current LEQ Experiments}

Fiber optic laser vibrometers are applied to obtain temporally-resolved particle velocity waveforms of the in-plane velocity components $\left(\dot{u}_{1}, \dot{u}_{2}\right)$ at fixed field points on the surface of the Homalite-100 test specimen. The three primary laser vibrometer configurations utilized for the experiments discussed in 75 this paper are depicted and briefly described in Figures $10(\mathrm{a}, \mathrm{b}, \mathrm{c})$. The outof-plane velocity component $\left(\dot{u}_{3}\right)$ may also be monitored as depicted in Figure 8.

Figure $10 \mathrm{~d}$ depicts a small $(3 \mathrm{~mm} \times 4 \mathrm{~mm})$ rectangular strip of retro-reflective tape with its upper left corner coincident with the field point $\left(\xi_{1}, \xi_{2}\right)$. Vibrom780 eter beams are focused down to a $150 \mu \mathrm{m}$ spot size onto a corner of the tape strip as shown. Scattered laser light is collected by each respective fiber-optic probe and directed by optical fiber to Polytec OFV-511 and OFV-551 heterodyne interferometers and an OFV-5000 modular controller (not depicted) where the interference signals are processed and converted into the recorded particle velocity waveforms. A common electrical trigger derived from the rupture nucleation circuit ensures temporal synchronization between the laser vibrometers 
and high-speed cameras. High-speed images showing the arrival of the propagating rupture front and its surrounding stress field at the measurement station are thus directly correlated to sharp jumps in the recorded particle velocity waveforms. Additional details of the electronic instrumentation, optical diagnostics, and LEQ experiment test procedures are contained in Mello et al. (2010).

\section{Results and Discussion}

\subsection{Investigation of Sub-Rayleigh and Supershear Rupture Velocity Waveform Signatures using the (FN:FP) Laser Vibrometer Configuration}

The high-speed images and corresponding particle velocity waveforms depicted in Figure 11 contrast the results obtained from two independent laboratory earthquake experiments, one of which yielded a right-lateral/left-traveling (rl/lt) sub-Rayleigh rupture and the other, which resulted in a (rl/lt) supershear rupture.

The position of the propagating rupture tip in the sub-Rayleigh rupture image (exp 0952), is identified by a dark, concentrated caustic zone positioned just behind the faint circular outline of the expanding $\mathrm{S}$ wavefront. In the supershear rupture image (exp 0947), the supershear rupture tip is flanked by shear Mach fronts, which extend to either side of the fault plane. An expanded view of the image reveals a localized fringe concentration, positioned just ahead of the supershear rupture tip, which coincides with the leading dilatational field lobe. A trailing sub-Rayleigh rupture with extended field lobes is also clearly visible and labeled in the image along with the circular outline of an expanding $\mathrm{S}$ wavefront.

The particle velocity waveforms obtained during the sub-Rayleigh and supershear experiments are each plotted out to $t=65 \mu \mathrm{s}$. The red triangles denote the time $(t=41 \mu s)$ when the displayed photoelastic images were acquired. Dashed blue vertical lines labeled $t_{p}$ and $t_{s}$ mark the anticipated arrival times of the nucleated $\mathrm{P}$ wave and $\mathrm{S}$ wavefronts at the measurement station located at $(-55,10) \mathrm{mm}$. Dashed blue vertical lines labeled $t_{s R}$ in each plot denote the arrival time of the sub-Rayleigh (sR) rupture in exp 0952 and the arrival time of the trailing sub-Rayleigh rupture in exp 0947. The dashed blue vertical line labeled $t_{\text {Mach }}$ in the supershear particle velocity record marks the anticipated arrival of the shear Mach front at the off-fault measurement station.

The synthetic curves, with the left and right panels, correspond to the particle velocity waveforms of right-lateral/left-traveling sub-Rayleigh and supershear ruptures, respectively, as predicted by the steady state singular elastic sub-Rayleigh shear crack solution. The time and velocity axes of the synthetic waveforms are dimensionless, and we make no attempt at a directly quantified comparison between theory and experiment. The primary aim is to demonstrate the strong correspondence between the form and polarity of the synthetic velocity waveforms and the corresponding particle velocity records obtained from each experiment. 

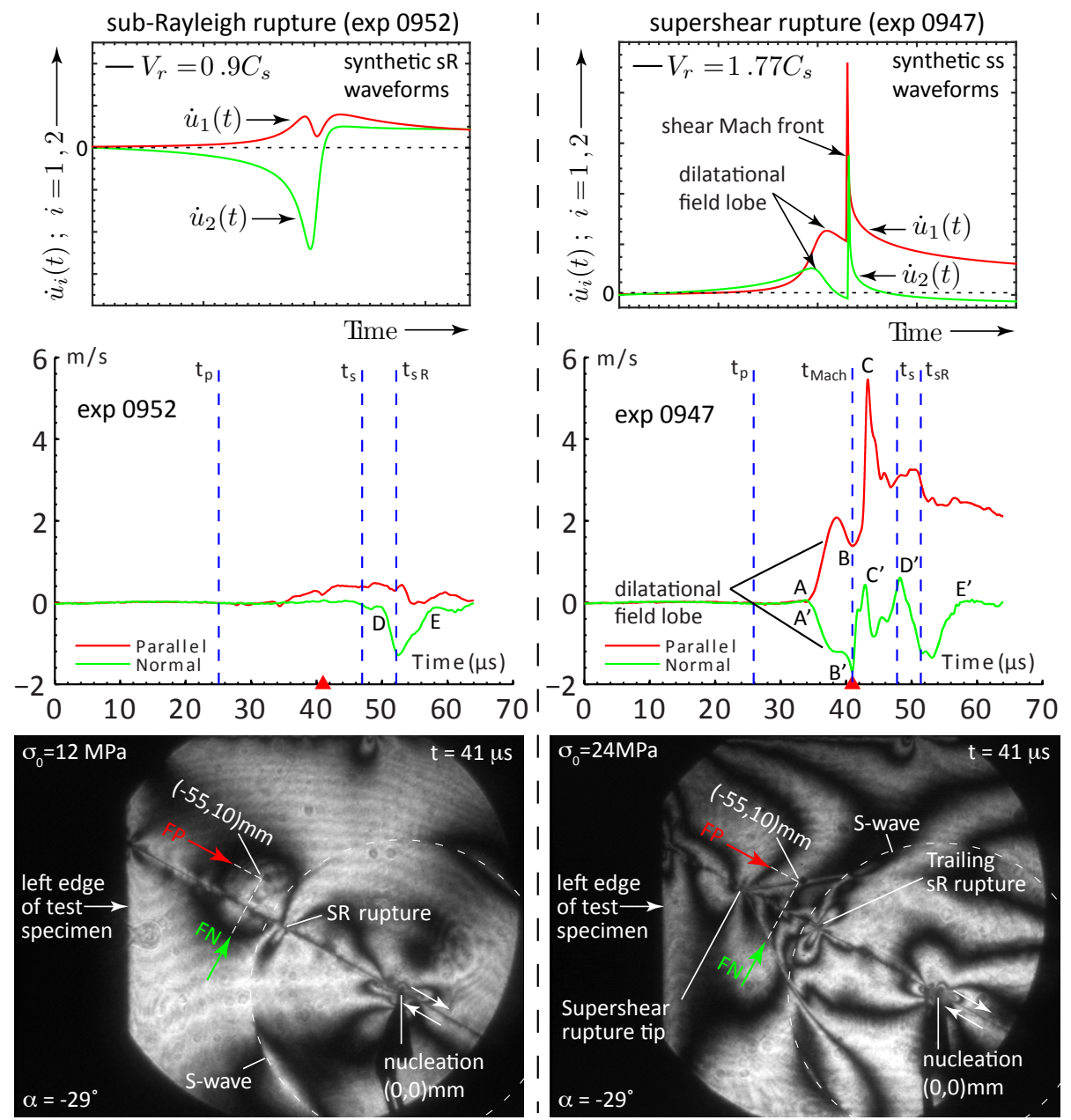

Figure 11: Left column (exp 0952): High-speed image of sub-Rayleigh rupture stress field at $t=41 \mu \mathrm{s}$, particle velocity waveforms acquired at $(-55,10) \mathrm{mm}$, and corresponding synthetic particle velocity waveforms derived from the steady state, singular elastic, (sub-Rayleigh) shear crack solution. Right column (exp0947): Dynamic rupture stress field at $t=41 \mu \mathrm{s}$, particle velocity waveforms acquired at $(-55,10) \mathrm{mm}$, and corresponding synthetic particle velocity waveforms from the steady state, singular elastic, (intersonic) shear crack solution.

Careful inspection of the photoelastic image sequence and particle velocity records obtained during the sub-Rayleigh rupture experiment (exp 0952) confirms that the segment $D \rightarrow E$ in the FN velocity record correlates with the time during which the extended sub-Rayleigh rupture field lobe swept through the off-fault measurement station at $(-55,10) \mathrm{mm}$. The experimental particle velocity records bear a strong correspondence with the form and polarity of the synthetic sub-Rayleigh waveforms and thus exhibit the distinct kinematic signatures induced by the upper field lobe of a (rl/lt) sub-Rayleigh rupture at this 

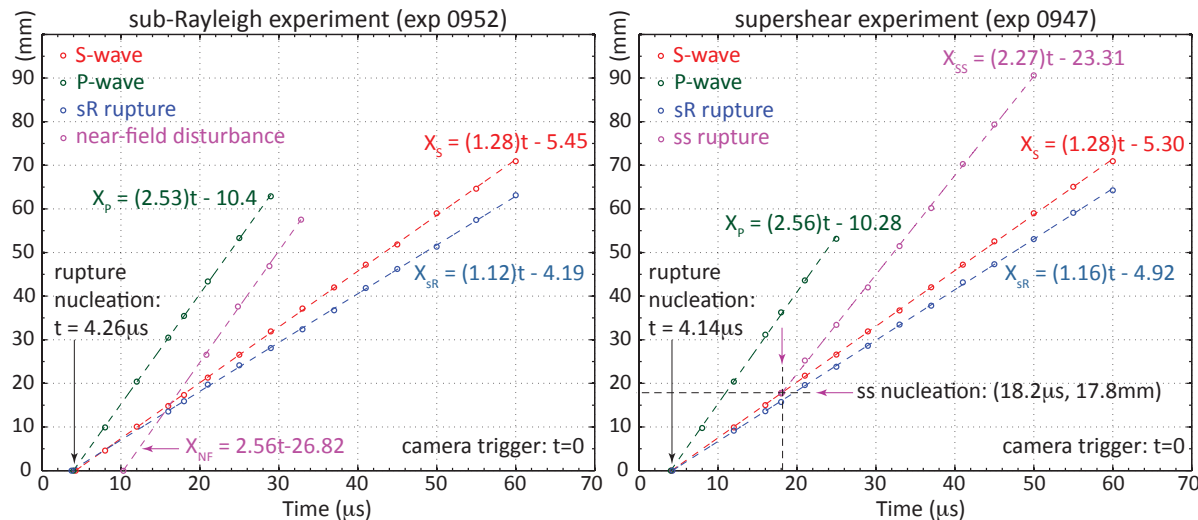

Figure 12: Distance-time plots of the rupture tip and elastic wavefronts obtained from photoelastic images for the sub-Rayleigh experiment (exp 0952) and supershear experiment (exp 0947). The intersection between the supershear rupture "best-fit line" and the S wave "best-fit line" provides a precise estimate of the location and time of the sub-Rayleigh to supershear transition in (exp 0947).

location. We also note the dominance of the FN component in the sub-Rayleigh velocity records as expected.

The FN and FP velocity records obtained during the supershear experiment 840 (exp 0947) embody distinct and fundamental waveform signatures, which are characteristic of a leading supershear rupture and trailing sub-Rayleigh rupture. The segment $A \rightarrow C$ of the FP component and $A^{\prime} \rightarrow C^{\prime}$ of the FN component in the ss particle velocity records bear an obvious and strong resemblance to the displayed synthetic waveforms derived from the steady state singular elastic 845 supershear crack solution. In particular, we note the positive initial velocity swing $A \rightarrow B$ of the $\mathrm{FP}$ component and sharp negative swing $A^{\prime} \rightarrow B^{\prime}$ of the FN component attributed to the leading dilatational field lobe as it swept the off-fault measurement station. This feature is immediately followed by the sharp positive velocity jumps, $B \rightarrow C$ in the FP component, and $B^{\prime} \rightarrow C^{\prime}$ in the FN component, commensurate with the arrival of shear Mach front. The finite rise time exhibited by the recorded supershear velocity jumps compared to the virtually instantaneous step-like jumps in the synthetic waveforms reflects the structure and spatial variation of the velocity field attributed to a finite rupture process zone.

The FP and FN velocity components records in (exp 0947) exhibit a precipitous drop following the passage of the shear Mach front and remain quiescent for a short period until the arrival of the trailing Rayleigh rupture coinciding with segment $D^{\prime} \rightarrow E^{\prime}$ in the FN waveform. We conclude by noting the dominance of the FN component during this time span and the strong resemblance between segment $D^{\prime} \rightarrow E^{\prime}$ and segment $D \rightarrow E$ in the FN waveform of the sub-Rayleigh experiment (exp 0952), as expected. 


\subsection{Experimental Estimates of the Average Rupture Speeds and Elastic Wave- speeds in Sub-Rayleigh and Supershear Rupture Experiments}

The plots displayed in Figure 12 represent position versus time curves of the rupture tip and elastic wavefronts obtained from sub-Rayleigh experiment (exp 0952) and supershear experiment (exp 0947). Distance is taken to be positive in the plots for the sake of convenience even though the measurement station was located at $(-55,10) \mathrm{mm}$. The colored circular symbols represent the measured distance from the nucleation site obtained through a frame-by-frame analysis of photoelastic images using well-documented procedures as outlined in Mello et al. (2010). Dashed lines passing through the circle symbols represent a best linear curve fit to the raw data. A linear equation describing the best-fit line is displayed beside each respective curve. The excellent linear fit observed in each case reveals how steady the elastic-wave and rupture speeds remained 875 throughout the duration of the experiments.

Analysis of the elastic wave speeds and rupture propagation speeds using the sub-Rayleigh high-speed images from exp 0952 yielded an estimated $\mathrm{P}$ wave speed of $C_{d}=2.53 \mathrm{~mm} / \mu \mathrm{s}$ (slope of the dashed green line) and $\mathrm{S}$ wave speed $C_{s}=1.28 \mathrm{~mm} / \mu \mathrm{s}$ (slope of the dashed red line). An average sub-Rayleigh rupture speed $\bar{V}_{r}=1.12 \mathrm{~mm} / \mu \mathrm{s}$, corresponding to $\bar{V}_{r} / C_{s}=0.88$, is obtained from the slope of the best fit line (dashed blue line). The dashed violet line labeled "near field" disturbance corresponds to a faint, expanding circular front, which is often seen in photoelastic images and propagates at the dilatational wave speed. Extrapolation of this curve back to the time axis reveals that the near field disturbance originated well after rupture nucleation, which suggests that it may be a release wave resulting from the dilatational contraction of the cylindrical cavity, which surrounds the $\mathrm{NiCr}$ wire.

A similar analysis applied to the photoelastic image sequence corresponding to the supershear experiment (exp 0947) yielded a P-wave speed estimate of $C_{d}=2.56 \mathrm{~mm} / \mu \mathrm{s}$, (slope of the dashed green line), and an S-wave speed estimate of $C_{s}=1.28 \mathrm{~mm} / \mu \mathrm{s}$, (slope of the dashed red line). An average subRayleigh rupture speed $\bar{V}_{r}=1.16 \mathrm{~mm} / \mu s$, corresponding to $\bar{V}_{r} / C_{s}=0.91$, is obtained from the slope of the best fit line (blue dashed blue line). We note the same sub-Rayleigh rupture speed was observed before and after the supershear transition. An average supershear rupture speed of $\bar{V}_{s s}=2.27 \mathrm{~mm} / \mu \mathrm{s}$, corresponding to $\bar{V}_{s s} / C_{s}=1.77$, is directly inferred from the slope of the best-fit line (dashed violet line). The intersection of the $\mathrm{S}$ wave curve and the supershear rupture curve at $(17.8 \mathrm{~mm}, 18.2 \mu \mathrm{s})$ provides a precise estimate of the location and time of the sub-Rayleigh to supershear transition.

The $\mathrm{S}$ wave, $\mathrm{P}$ wave, and sub-Rayleigh rupture curves in Figure 12 extrapolate back to a set of closely spaced points, which intercept the time axis. The time axis intercepts reveal an inherent rupture delay time between the instrumentation trigger $(t=0)$ (oscilloscope and high-speed camera are triggered), and the time at which elastic waves were first emitted from the rupture nucleation site. The noted time delay is repeatedly observed in LEQ experiments and is related to the rupture nucleation process, as opposed to being an artifact of 


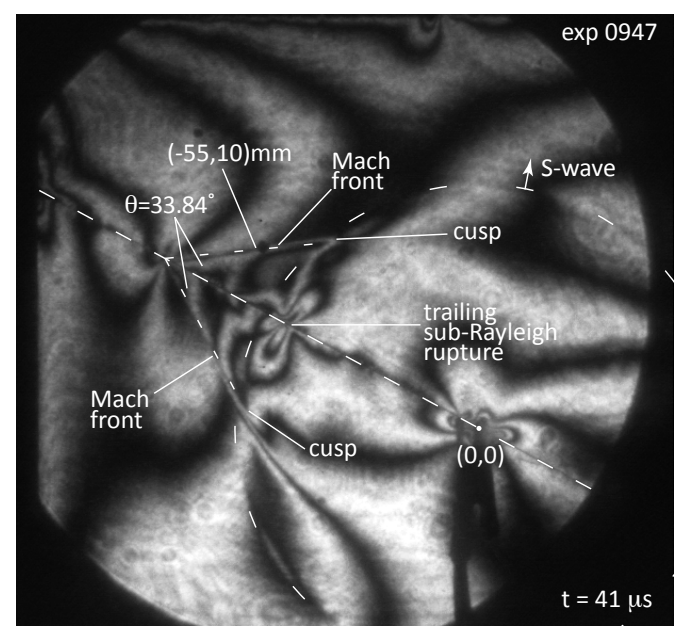

Figure 13: Expanded view of supershear rupture field with measured Mach front angles for determination of instantaneous supershear rupture speed.

the electronic diagnostics. The time axis intercept for the sub-Rayleigh rupture curve is not physically meaningful since the rupture tip is never located at the origin, but is instead initiated at a critical-crack-length distance located to the left and right of the origin. Time axis intercepts derived from the P-wave curves are unreliable due to a greater uncertainty of the P-wave speed. The rupture delay time is best determined from the $\mathrm{S}$ wave time axis intercept, and is always taken into account when determining the elastic wave arrival times with respect to the rupture arrival times in the recorded particle velocity waveforms.

\subsection{Determination of the Instantaneous Supershear Rupture Speed from Mach} Angle Measurements

Figure 13 corresponds to an expanded view of the photoelastic image acquired at time $t=41 \mu \mathrm{s}$ during the supershear experiment (exp 0947). An independent estimate of the instantaneous supershear rupture speed is obtained by measuring the shear Mach angle and applying the familiar Mach cone halfangle relation Eq. 16, which is restated here for the sake of convenience.

$$
\sin \theta=C_{s} / V_{s s}
$$

Sharp cusps in the fringe pattern, such as those labeled in Figure 13 reveal the location of the steepest stress gradient and thus provide a natural fiducial for extending a line from the rupture tip when estimating the Mach angle. Lines ${ }_{925}$ were drawn from the supershear tip to each of the sharp cusps highlighted in Figure 13 to determine the shear Mach angle. Mach angle measurements of $\theta_{1}=33.75^{\circ}$ and $\theta_{2}=33.97^{\circ}$ were obtained. A measurement uncertainty of $\pm 0.25^{\circ}$ in the measurement of the Mach angle translates to an error bound of \pm 0.01 in the normalized rupture speed estimate. Application of Eq. 34 to the average value of these two values yields an instantaneous normalized rupture 
speed estimate of $V_{s s} / C_{s}=1.79 \pm 0.01,\left(V_{s s}=2.29 \pm 0.01 \mathrm{~mm} / \mu \mathrm{s}\right)$, which is in excellent agreement with the average supershear rupture speed estimate $\left(V_{r} / C_{s}\right)_{\text {ave }}=1.77$ as determined from the slope of the best-fit line in Figure 12

\subsection{High-speed Images and Particle Velocity Signatures of an Emerging Su-} pershear Daughter Crack Propagating at $V_{r}=\sqrt{2} C_{s}$ and the Onset of a supershear Transition.

The photoelastic image sequence and particle velocity records displayed in Figure 14 bear the collective signatures of an emerging supershear daughter crack and the onset of a sub-Rayleigh to supershear transition. The supershear rupture is trailed by a "crack-like" sub-Rayleigh (sR) rupture, which correlates with the large amplitude swings in the recorded particle velocity records.

A magnified view of the image frame recorded at $t=49 \mu \mathrm{s}$ (Figure 14(a)), reveals a dark, curled fringe (labeled in the image), which appears to terminate at the fault plane. The magnified image also reveals a dark, straight fringe concentration, aligned with the fault, which juts out from the sub-Rayleigh rupture tip and extends out to the circular boundary of the leading $\mathrm{S}$ wavefront. These 2 noted features within the photoelastic interference pattern reveal an emerging supershear daughter crack and signal the onset of a Burridge-Andrews supershear transition Mello et al. (2010). The point where the dark curled 950 fringe intersects the fault plane coincides with the leading tip of the supershear daughter crack. The trailing, dark fringe concentration aligned with the fault is a precursor to the shear Mach fronts, which form at a later time.

Calibrated distance measurements of the daughter crack positions in the sequential image frames recorded at $t=49 \mu \mathrm{s}$ (Figure 14(a)) and $t=53 \mu \mathrm{s}$ (Figure 14(b)) yield a relative distance of $\Delta x_{1}^{s s}=7.33 \mathrm{~mm}$, which translates to an average normalized rupture speed of $V_{r} / C_{s}=1.43$ during the $4 \mu s$ time interval. The other noted fringe concentration, which is aligned with the fault, and trails the daughter crack tip, has transformed at $t=53 \mu \mathrm{s}$ and provides an early hint of the shear Mach fronts, which are just beginning to form at this 960 time. The image frame recorded at $t=57 \mu s$ (Figure 14(c)), reveals the rapidly accelerating supershear rupture tip flanked by shear Mach fronts, which are now quite evident. Analysis of the supershear crack tip positions in the sequential image frames recorded at $t=57 \mu \mathrm{s}$ and $t=60 \mu \mathrm{s}$ reveals a relative distance of $\Delta x_{1}^{s s}=7.08 \mathrm{~mm}$, which translates to an average normalized rupture speed of $V_{r} / C_{s}=1.84$ during the $3 \mu \mathrm{s}$ time interval. Measurement of the shear Mach angle in the image recorded at $t=60 \mu \mathrm{s}$ yields an instantaneous normalized rupture speed estimate of $V_{r} / C_{s}=1.83$, which agrees very well with the average normalized rupture speed measurement. Calibrated distance measurements of the sub-Rayleigh crack tip position in the sequential images recorded at $t=49 \mu \mathrm{s}$ 970 and $t=57 \mu \mathrm{s}$ yield a relative distance of $\Delta x_{1}^{s R}=9.14 \mathrm{~mm}$, which translates to an average normalized rupture speed of $V_{r} / C_{s}=0.89$ during the $8 \mu s$ time interval. The supershear rupture has now fully transitioned and is propagating at $2 \times$ the speed of the trailing sub-Rayleigh rupture from which it was spawned.

We now focus our attention on the analysis and interpretation of the FP and FN particle velocity records depicted in Figure $14(\mathrm{e})$. The noted $4 \mu s$ pulse-like 

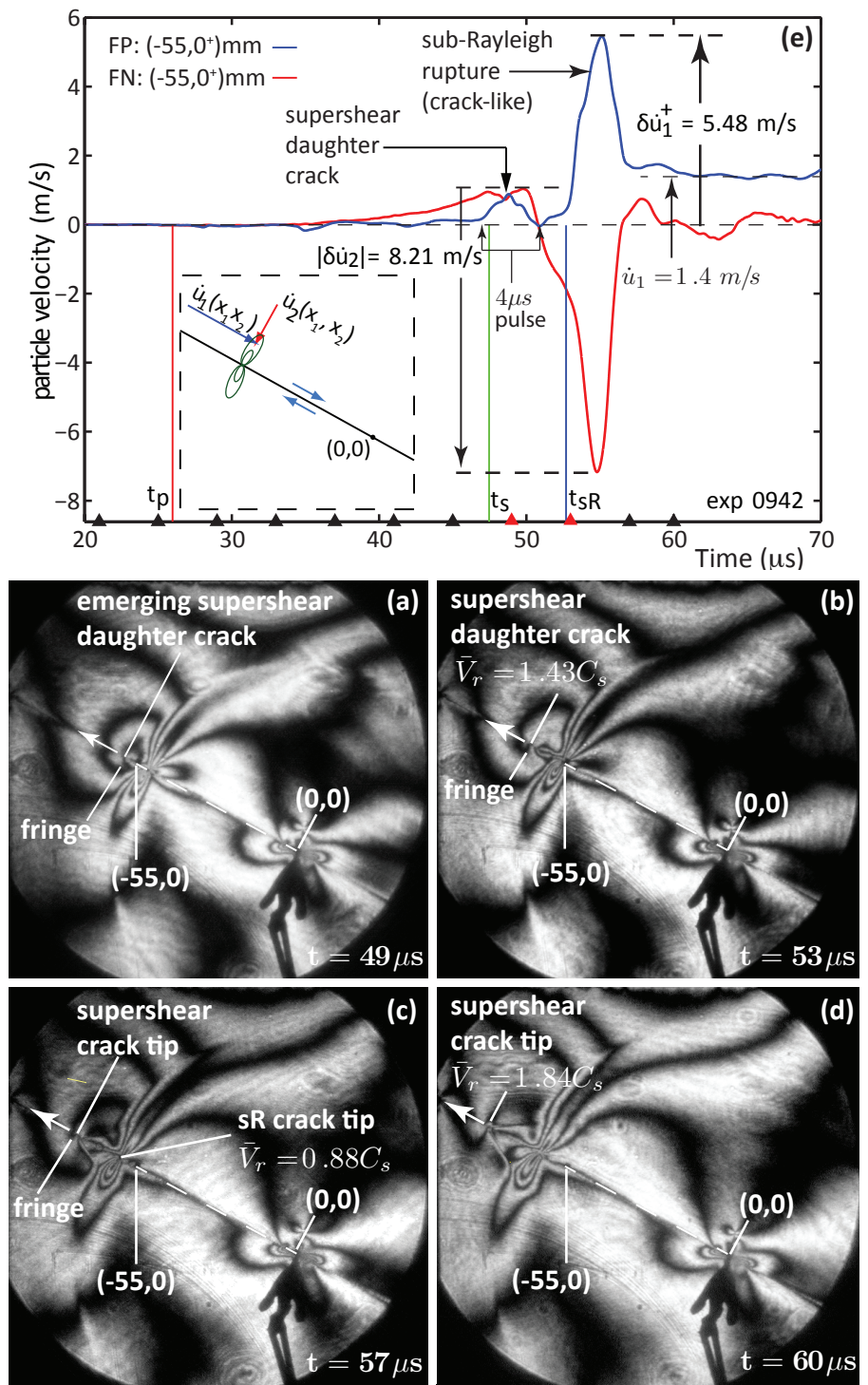

Figure 14: (a,b) Supershear daughter crack with average rupture speed $\bar{V}_{r}=1.43 \mathrm{~mm} / \mu \mathrm{s}$ between the 2 sequential image frames. (c,d) Supershear crack with average rupture speed $\bar{V}_{r}=1.84 \mathrm{~mm} / \mu \mathrm{s}$ between the 2 sequential image frames. (e) Particle velocity records recorded at $\left(-55,0^{+}\right)$reveal a pulse-like disturbance in the $\mathrm{FP}$ component attributed to the emerging supershear daughter crack crossing the on-fault station at $V_{r}=\sqrt{2} C_{s}$. This is followed by the signature crack-like rupture velocity profile of the trailing sub-Rayleigh rupture.

disturbance in the FP velocity record is attributed to the emerging supershear daughter crack, which swept the near-fault station, between $t=47 \mu \mathrm{s}$ and $t=51 \mu \mathrm{s}$, well in advance of the sub-Rayleigh rupture. The positive velocity jumps exhibited by the FN and FP velocity components during the noted pulse 

propagating with a stable supershear rupture speed $\left(V_{r} \geq \sqrt{2} C_{s}\right)$ as it crossed the on-fault station. This observation, coupled with the average normalized rupture speed estimate of $V_{r}=1.43 C_{s}$ observed between the image frames recorded at $t=49 \mu \mathrm{s}$ and $t=53 \mu \mathrm{s}$, conservatively bound the rupture speed interval to surement the conclusion that it was propagating with a rupture speed much closer to $\sqrt{2} C_{s}$ as it crossed the measurement station between $t=47 \mu \mathrm{s}$ and $t=51 \mu \mathrm{s}$. The conspicuous absence of any shear Mach fronts in the synchronized image recorded at $t=49 \mu \mathrm{s}$ only serves to bolster this hypothesis. The positive velocity swings exhibited by each waveform component during the noted $4 \mu \mathrm{s}$ pulse period are evidently attributed to the dilatational field, which circulates about the supershear rupture tip, as depicted in Figure 4 .

The general profile and polarity corresponding to the sub-Rayleigh portion of of motion of a right-lateral/left-traveling sub-Rayleigh rupture. The particle velocity waveforms are characterized by a dominant $\mathrm{FN}$ component, as expected for a sub-Rayleigh rupture, with the ratio between the velocity components given by $\left|\delta \dot{u}_{2}^{+}\right| /\left|\delta \dot{u}_{1}^{+}\right|=(8.21 \mathrm{~m} / \mathrm{s}) /(5.48 \mathrm{~m} / \mathrm{s})=1.5$. The resulting particle velocity

1000 waveforms bear the distinct signature of a "crack-like" sub-Rayleigh rupture, as revealed by the nearly constant sliding speed of $\dot{u}_{1}^{+}=1.4 \mathrm{~m} / \mathrm{s}$, which was sustained well after the rupture had crossed the measurement station.

The sub-Rayleigh rupture velocity waveforms gathered in (exp 0942) are representative of a broad range of sub-Rayleigh rupture experiments, which were conducted using the (FN:FP) laser vibrometer configuration. The dominance of the FN component was observed in all cases, i.e $\left|\delta \dot{u}_{2}\right| /\left|\delta \dot{u}_{1}\right|>1$, without exception. The form and polarity exhibited by sub-Rayleigh particle velocity waveforms in our experiments are always found to be consistent with the anticipated sense of particle motion, as predicted by theory.

\subsection{Investigation of the Velocity Component Ratio $\delta \dot{u}_{1}^{s}\left(z_{s}\right) / \delta \dot{u}_{2}^{s}\left(z_{s}\right)$ along a} Shear Mach Front using the (FN:FP) Laser Vibrometer Configuration

A set of supershear rupture experiments were conducted using the (FP:FN) laser vibrometer probe configuration depicted in Figure 10 (a). Simultaneous particle velocity waveforms were recorded at a variety of off-fault field points, as a supershear rupture and its surrounding field swept past the measurement station. Direct measurement of the particle velocity jumps $\delta \dot{u}_{1}^{s}\left(z_{s}\right)$ and $\delta \dot{u}_{2}^{s}\left(z_{s}\right)$ along the shear Mach front were obtained by taking advantage of the increased spatiotemporal separation between the leading dilatational field lobe and the shear Mach front, with increased FN distance from the fault plane. The ratio $\beta_{s}^{\text {meas }}=\delta \dot{u}_{1}^{s}\left(z_{s}\right) / \delta \dot{u}_{2}^{s}\left(z_{s}\right)$ was determined for 10 independent supershear experiments conducted over a broad range of stable supershear rupture speeds $\left(\sqrt{2} C_{s}<V_{r}<C_{d}\right)$. Results were then plotted against the normalized rupture speed $\left(V_{r} / C_{s}\right)$, which was determined through direct measurement of the shear Mach front angle as previously outlined. 

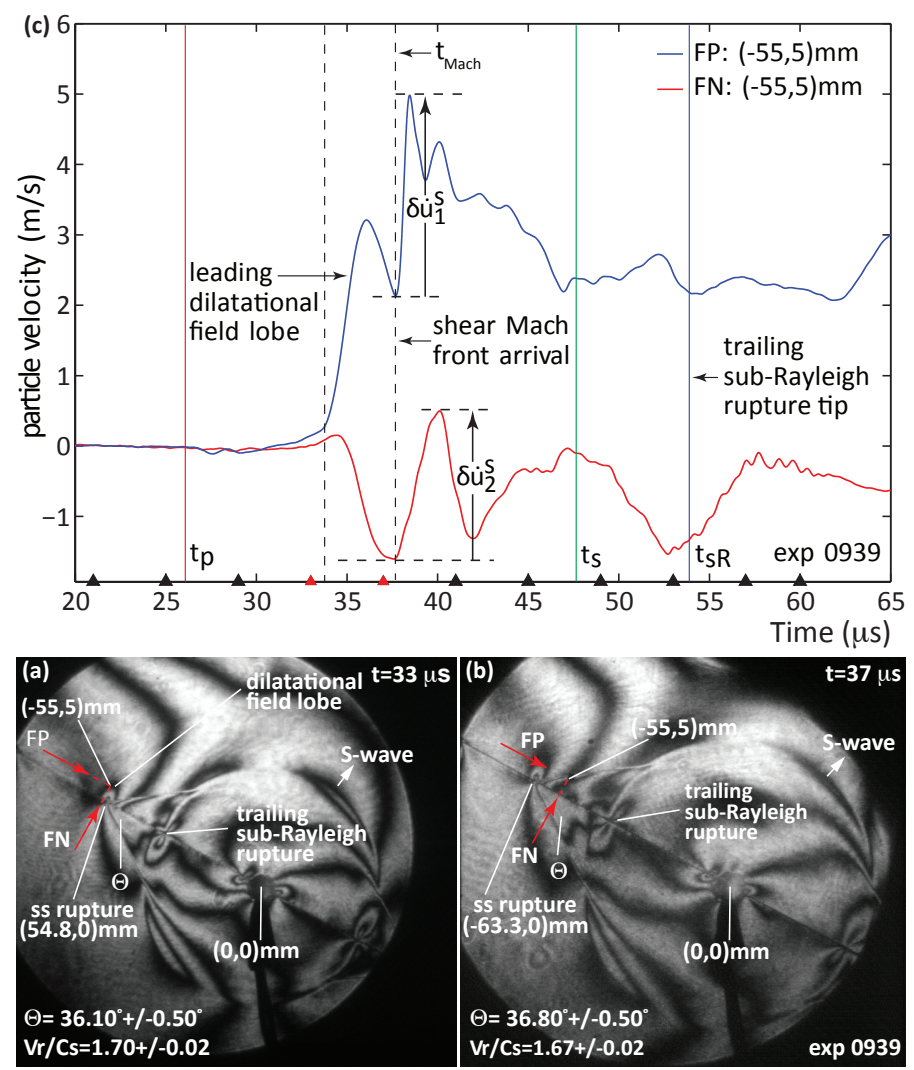

Figure 15: (a,b) Consecutive photoelastic image frames capture a shear Mach front crossing a particle velocity measurement station positioned at $(-55,5) \mathrm{mm}$. (c) Simultaneous FN and FP particle velocity records bear the distinct signatures of a leading dilatational field lobe followed by the sharp velocity jump linked to the arrival of the shear Mach front, as portrayed by the synthetic waveforms displayed in Figure 11

Figure 15 summarizes the key results obtained from a representative supershear rupture experiment (exp 0939). The consecutive high-speed image frames in Figure 15(a,b) reveal the supershear rupture and its surrounding field as it approached and subsequently swept the off-fault measurement station located at $(-55,5) \mathrm{mm}$. Figure 15 (a), recorded at $t=37 \mu \mathrm{s}$, shows the dilatational field lobe just as it began to sweep the measurement station. Figure 15 (b) reveals the shear Mach front just as it reached the measurement station at $t=37 \mu \mathrm{s}$.

Figure 15. displays the synchronized FN and FP particle velocity records recorded at the off-fault station $(-55,5) \mathrm{mm}$. The pronounced velocity jumps $\delta \dot{u}_{1}^{s}\left(z_{s}\right)$ and $\delta \dot{u}_{2}^{s}\left(z_{s}\right)$ attributed to the arrival of the shear Mach front are anno1035 tated in the figure. We note a $1.35 \mu s$ offset between the corresponding peaks of the FP and FN particle velocity records in (exp 0939). The observed offset stems from the longer rise time exhibited by $\delta \dot{u}_{2}^{s}\left(z_{s}\right)$ in the FN record. The cause of this unusually long rise time is not well understood. One possible ex- 


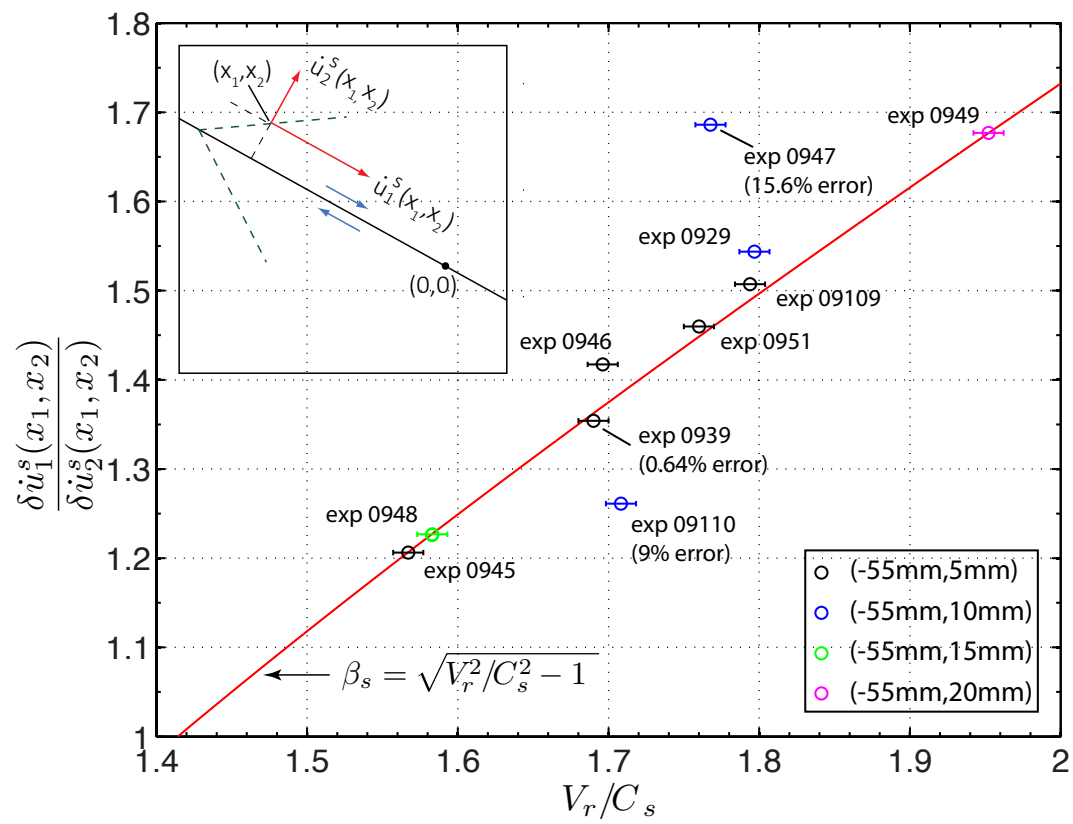

Figure 16: Measurements of $\delta \dot{u}_{1}^{s} / \delta \dot{u}_{2}^{s}$ determined from supershear experiments plotted versus the normalized rupture speed $V_{r} / C_{s}$. Excellent agreement between theory and experiment is demonstrated across a broad range of rupture speeds, which nearly span the entire domain of the supershear speed regime.

planation is that the maximum slew rate of the amplified signal was reduced due to an incorrect (lowered) electronic bandwidth setting of the modular controller channel dedicated to the FN vibrometer signal. A measured velocity jump of $\delta \dot{u}_{1}^{s}=2.844 \mathrm{~m} / \mathrm{s}$ in the FP record and a corresponding velocity jump of $\delta \dot{u}_{2}^{s}=2.101 \mathrm{~m} / \mathrm{s}$ exhibited by the FN signal leads to an experimental estimate of $\beta_{s}^{\text {meas }}=\delta \dot{u}_{1}^{s} \mid / \delta \dot{u}_{2}^{s}=1.354$ for the ratio between the FP and FN jumps along the shear Mach front. The measured value may be compared to the theoretically predicted value given by Eq. 22 i.e., $\beta_{s}=\frac{\delta \dot{u}_{1}^{s}}{\delta \dot{u}_{2}^{s}}=\sqrt{V_{r}^{2} / C_{s}^{2}-1}$ assuming that the normalized supershear rupture speed is known. An estimate of the instantaneous supershear rupture speed was obtained by measuring the acute angles subtended by the shear Mach fronts in both image frames. The measured value of $V_{r} / C_{s}=1.69 \pm 0.02$ agrees very well with the average normalized rupture speed $\bar{V}_{r} / C_{s}=1.66$ obtained through a frame-by-frame analysis of the rupture tip position spanning 3 image frames recorded between $t=29 \mu \mathrm{s}$ and $t=37 \mu \mathrm{s}$. The measured normalized rupture speed translates to a theoretically predicted value of $\beta_{s}=1.36 \pm 0.03$ in accordance with Eq 10 . The measured value of $\beta_{s}^{\text {meas }}=1.354$ is thus found to be within $1 \%$ of the theoretically predicted value.

The supershear experiment (exp 0939) summarized in Figure 15 is representative of a group of 10 similar supershear experiments conducted over a broad 
range of rupture speeds, which were analyzed in a similar manner. Estimates for $\beta_{s}^{\text {meas }}$ obtained from each of these experiments are plotted against the corresponding (measured) normalized rupture speed in Figure 16 . The solid red curve in the plot corresponds to the theoretical value given by $\beta_{s}=\sqrt{V_{r}^{2} / C_{s}^{2}-1}$. The individual data points are color coded according to the FN distance $\left(x_{2}\right)$ of the measurement station in the experiment. The horizontal error bars in Figure 16

1065 correspond to the measurement uncertainty of \pm 0.01 in the normalized rupture speed estimate.

Eight out of the ten supershear experiments represented in Figure 16 yielded a value for $\beta_{s}^{\text {meas }}$ that was found to be within $3.44 \%$ of the theoretically predicted value. There are two noted "outliers", in the plot corresponding to a $9 \%$ error in (exp 09110) and $15.6 \%$ error observed in (exp 0939). The larger error observed in these two experiments is attributed to the "near field disturbance" discussed in section 8.2. which was superimposed upon the supershear rupture field as the shear Mach front swept the off-fault station. The commonality between the eight "known good" experiments is that they all exhibited an early sR to SS rupture transition, which allowed the shear Mach front to sweep the measurement in advance of the near field disturbance. Experiments featuring early sR to SS rupture transitions are essential to achieving reliable shear Mach front measurements when using the electrical rupture nucleation scheme.

Bearing all of these points and observations in mind, we conclude that the experimental results summarized by the plot in Figure 16 demonstrate excellent agreement between theory and measurement. The results demonstrate that the observed ratio between the magnitudes of the FP and FN components along the shear Mach front is governed by the theoretical relationship given by Eq. 22. The relationship is preserved out to a considerable distance from the fault 1085 plane, approximately $2 \times$ the thickness of the slipping region. In natural faults, this would correspond to several 10's of kilometers.

\subsection{Amplitude Decay of sub-Rayleigh Rupture Fields: Experimental Investiga- tions using the (FN1:FN2) Vibrometer Configuration}

A set of sub-Rayleigh rupture attenuation experiments were conducted using the (FN1:FN2) laser vibrometer configuration depicted in Figure 10b. FN particle velocity records were simultaneously recorded at an on-fault measurement station $\left(x_{1}, 0^{+}\right)$and off-fault measurement station $\left(x_{1}, x_{2}\right)$ as a sub-Rayleigh rupture and its extended field lobes swept past these locations. The amplitude decay of the sub-Rayleigh rupture velocity field was investigated by examining the ratio $\left|\delta \dot{u}_{2}\left(x_{1}, x_{2}\right)\right| /\left|\delta \dot{u}_{2}\left(x_{1}, 0^{+}\right)\right|$of the corresponding velocity swings at each location and plotting this ratio as a function of the fault normal distance $\left(x_{2}\right)$. The primary goal of the study was to investigate whether the observed amplitude decay was governed by a $1 / \sqrt{r}$ relationship as predicted by the singular elastic dynamic shear crack model and to note any observed departures from 1100 this theoretical prediction.

Figure 17 summarizes the key measurements obtained from a representative sub-Rayleigh rupture experiment (exp 0973). A rupture arrival time estimate of $t=51.88 \mu \mathrm{s}$, corresponding to a normalized rupture speed of $V_{r} / C_{s}=0.91$, 

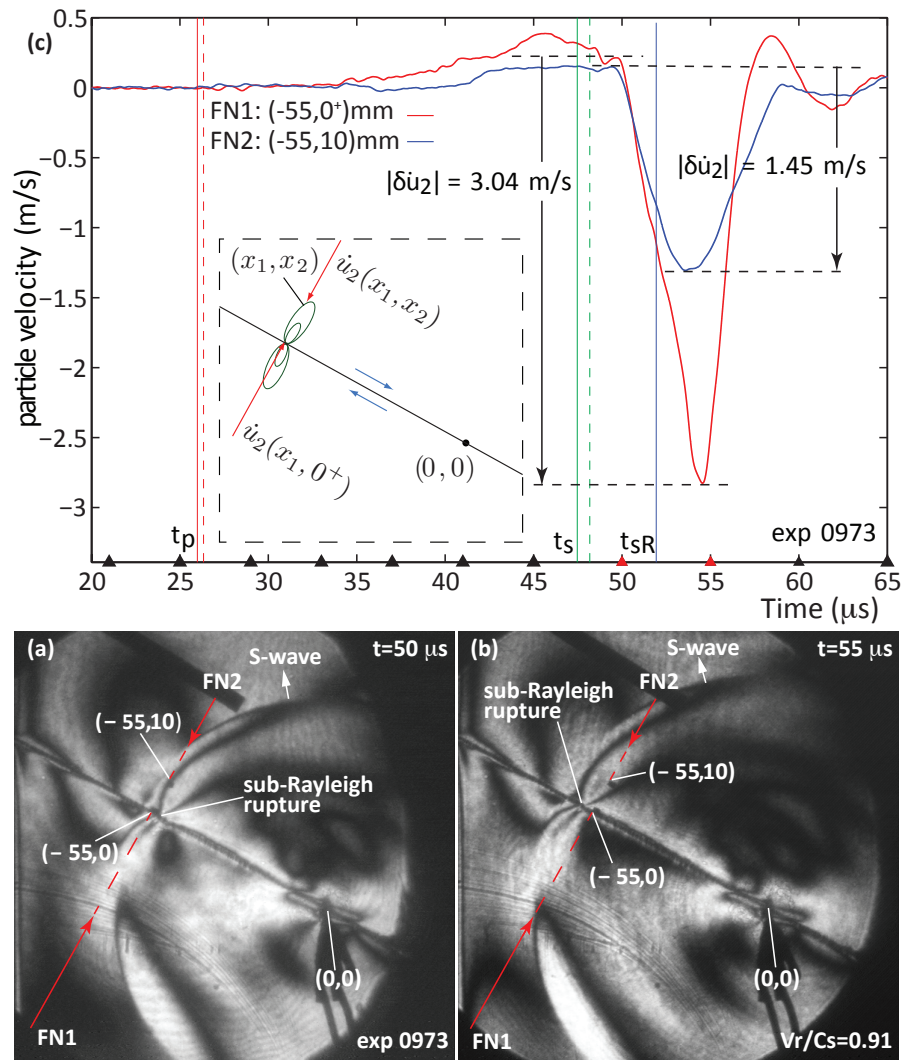

Figure 17: $(\mathrm{a}, \mathrm{b})$ Consecutive photoelastic image frames reveal the sub-Rayleigh rupture and its surrounding stress field lobes as they approach and subsequently cross the particle a pair of measurement stations located at $(-55,0) \mathrm{mm}$ and $(-55,10) \mathrm{mm}$. (c) FN particle velocity traces simultaneously recorded at the on-fault and off-fault stations obtained by a (FN1:FN2) vibrometer probe configuration as depicted in the inset. The FN record obtained at $(-55,10) \mathrm{mm}$ has decayed to $48 \%$ of its corresponding on-fault value.

was determined through a frame-by-frame analysis of the high-speed images. The noted rupture speed observed in (exp 0973) is representative of the range of normalized rupture speeds $0.88 \leq V_{r} / C_{s} \leq 0.91$ observed in this study.

The pair of photoelastic image frames acquired at $t=50 \mu \mathrm{s}$ and $t=55 \mu \mathrm{s}$ in Figures 17(a,b) reveal a sub-Rayleigh rupture field as it approached and subsequently swept the on-fault and off-fault particle velocity measurement stations 1110 located at $\left(-55,0^{+}\right) \mathrm{mm}$ and $(-55,10) \mathrm{mm}$, respectively. Figure $17 \mathrm{a}$, acquired at $t=50 \mu \mathrm{s}$, depicts the sub-Rayleigh rupture tip as it approached the on-fault measurement station $\left(-55,0^{+}\right) \mathrm{mm}$ while the leading edge of the extended field lobe approached the off-fault station at $(-55,10) \mathrm{mm}$. Figure $17 \mathrm{~b}$, acquired at $t=55 \mu \mathrm{s}$, reveals the rupture tip just after it crossed the on-fault station while the trailing edge of the extended field lobe is seen crossing the off-fault station. The FN velocity swings observed in (exp 0973) are each annotated and labeled 
Amplitude Decay of sub-Rayleigh Ruptures

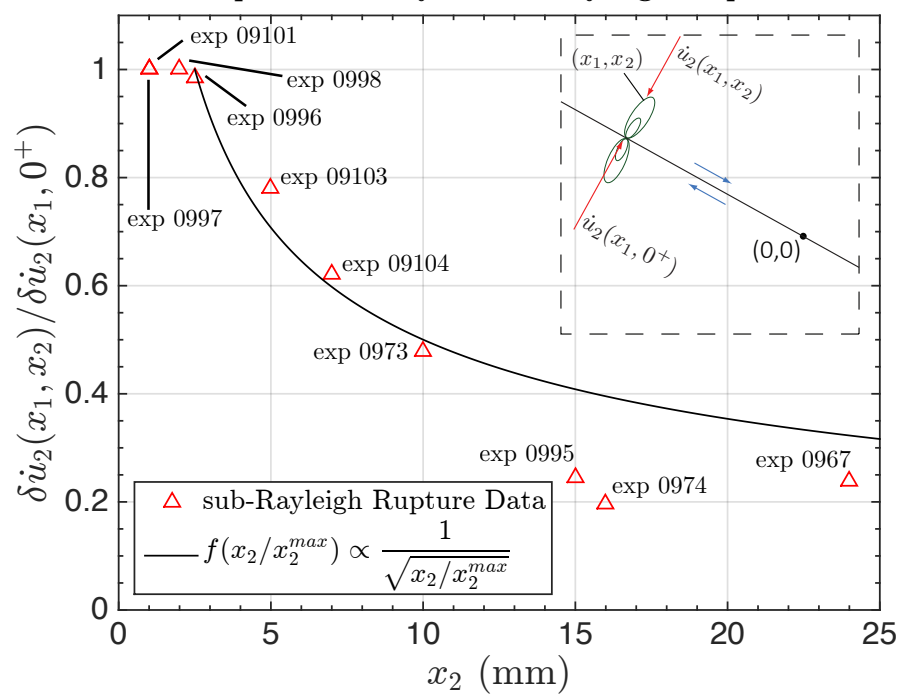

Figure 18: Amplitude decay of a sub-Rayleigh particle velocity field revealed by the measured ratio $\delta \dot{u}_{2}\left(x_{1}, x_{2}\right) / \delta \dot{u}_{2}\left(x_{1}, 0^{+}\right)$plotted versus the FN distance $x_{2}$. Results were obtained using the (FN1:FN2) vibrometer probe configuration depicted in the inset. The normalized curve $f\left(x_{2} / x_{2}^{\max }\right) \propto 1 / \sqrt{x_{2} / x_{2}^{\max }}$ provides a reasonable fit of the data points spanning the range $\left(2.5 \mathrm{~mm}<x_{2}<10 \mathrm{~mm}\right)$.

in Figure 17. Measured velocity swings of $\delta \dot{u}_{2}=3.04 \mathrm{~m} / \mathrm{s}$ at $\left(-55,0^{+}\right) \mathrm{mm}$ and $\delta \dot{u}_{2}=1.45 \mathrm{~m} / \mathrm{s}$ at the off-fault field point $(-55,10 \mathrm{~mm})$ translate to an amplitude decay of $48 \%$ at a distance $x_{2}=10 \mathrm{~mm}$ from the fault plane.

Experiment (exp 0973) is representative of 10 independent dynamic rupture experiments, which were all monitored using the (FN1:FN2) laser vibrometer configuration. Figure 18 summarizes the results of these experiments by plotting the normalized amplitude decay $\delta \dot{u}_{2}\left(x_{1}, x_{2}\right) / \delta \dot{u}_{2}\left(x_{1}, 0^{+}\right)$versus the FN distance $\left(x_{2}\right)$. Note that although only nine symbols are shown, there are two superimposed triangular symbols corresponding to the data point at $x_{2}=1 \mathrm{~mm}$ and so there are actually ten experiments represented in the plot. The cluster of data points stationed within $\left(1 \mathrm{~mm} \leq x_{2} \leq 2.5 \mathrm{~mm}\right)$ with an amplitude decay ratio near unity are a direct manifestation of the rupture process zone. The slight drop in the normalized amplitude decay to a value of 0.986 at $x_{2}=2.5 \mathrm{~mm}$ signals the

1130 boundary of this zone, beyond which the amplitude ratio is observed to rapidly decay. A normalized curve of the form $f\left(x_{2} / x_{2}^{\max }\right) \propto 1 / \sqrt{x_{2} / x_{2}^{\max }}$ provides a reasonable fit of the data points spanning the range $\left(2.5 \mathrm{~mm}<x_{2}<10 \mathrm{~mm}\right)$ but tends to significantly overpredict the amplitude decay values observed at $x_{2}=15 \mathrm{~mm}$ and $x_{2}=24 \mathrm{~mm}$. 


\subsection{Relationship Between the Rupture Sliding Speed and the FP Velocity Com- ponent Propagated by a Shear Mach Front: Experimental Investigations using the (FP1:FP2) Vibrometer Configuration}

The particle velocity waveforms in Figure 19(a) were obtained from 4 independent supershear rupture experiments. In each case, a FP vibrometer probe beam was focused at a different off-fault field point as indicated. The peak velocity attained by the leading dilatational field lobe and shear Mach front portions of each velocity record are labeled $p$ and $q$, respectively. The progressive separation $\Delta t_{p q}$ between these two peaks reveals how rapidly the two fields begin to separate with increased distance $\left(x_{2}\right)$ from the fault plane. Indeed, this very same field property was exploited in order to obtain the experimental estimates of $\beta_{s}^{\text {meas }}=\dot{u}_{1}^{s} / \dot{u}_{2}^{s}$ summarized in Figure 16 . Another striking feature revealed in the experimental records is the pronounced broadening and rapid amplitude decay of the dilatational field lobe, which is in stark contrast to the sharper and more sustained velocity jump propagated by the shear Mach front.

In actuality, the experiments depicted in Figure 19(a) were each characterized by unique stress drops, supershear transition distances, and rupture speeds, and so the stacked velocity records are not equivalent to four simultaneous FP measurements obtained from a single supershear experiment. The ideal experiment to demonstrate this effect would require a set of simultaneous FP measurements conducted at different distances from the fault plane during a single supershear rupture experiment. A limited number of laser vibrometers prohibited this approach, and so the results from 4 independent experiments were collated to create as shown to visualize the spatiotemporal separation between the shear Mach fronts and the dilatational field lobe.

Figure 19(b) portrays a corresponding set of synthetic velocity records derived from the steady state singular elastic shear crack model. As previously noted, the time and velocity axes of the synthetic waveforms are dimensionless, and we make no attempt to conduct a directly quantified comparison between theory and experiment. The aim is to highlight universal spatiotemporal properties, which emerge from the steady state dynamic rupture models such as singular elastic shear crack model and note the presence of these very same features within our experimental particle velocity records. As such, there is a striking similarity between the spatiotemporal features exhibited by the synthetic waveforms and those observed in the experimental particle velocity records. In particular, we note the progressive decay of the dilatational field and the increased separation between the dilatational field lobe and the shear Mach front with increased distance $\left(x_{2}\right)$ from the fault plane. The slower rise time of the velocity jumps associated with the arrival of the shear Mach front in the experimental records compared to the virtually instantaneous step-like velocity jumps predicted by the steady state singular model, reflects a spatial variation of the velocity field within the shear Mach cone attributed to a finite rupture process zone.

A set of supershear rupture experiments were conducted using the (FP1:FP2) vibrometer configuration (Figure $10(\mathrm{a})$ ) in order to investigate the scaling re- 


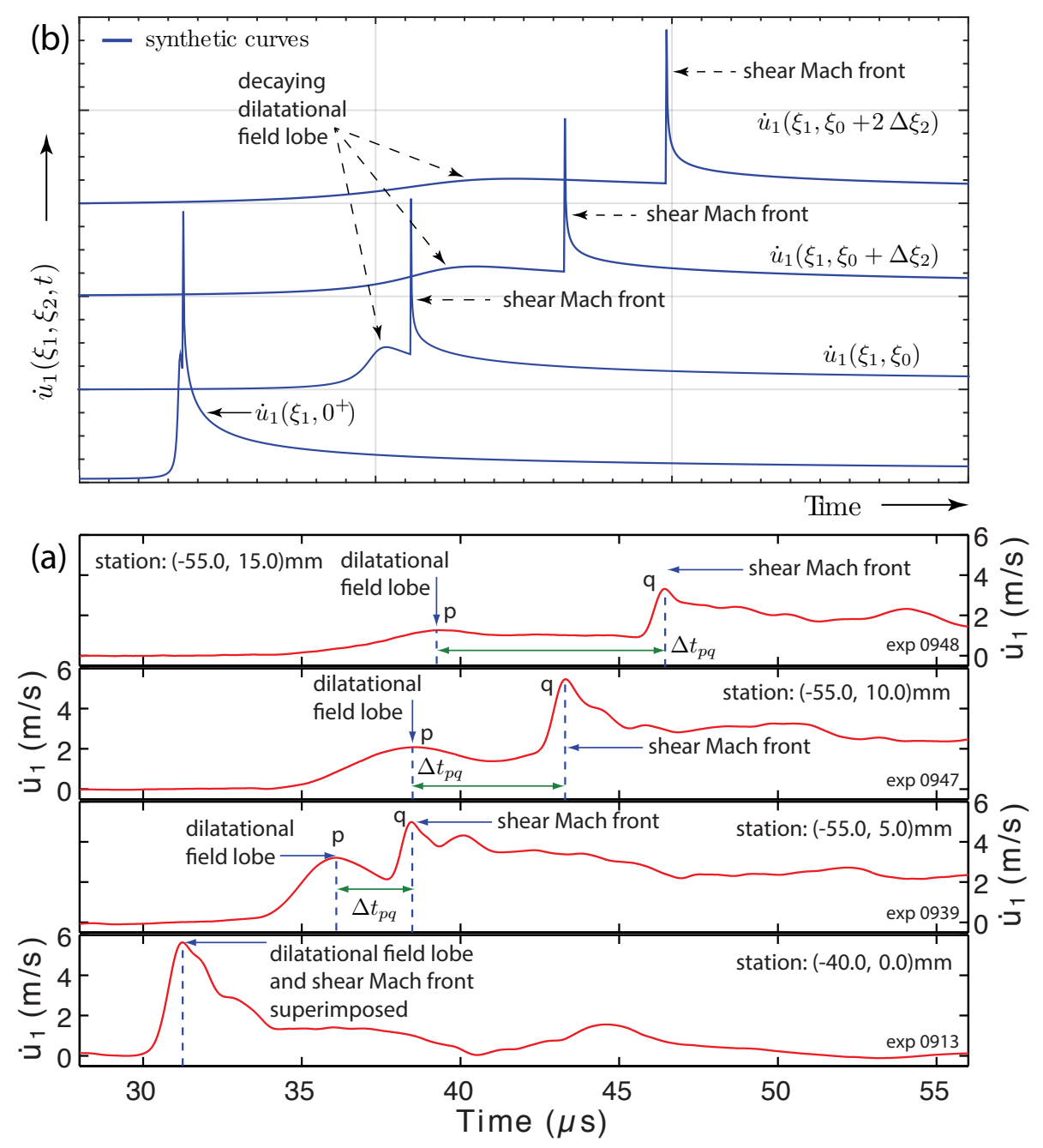

Figure 19: Synthetic curves derived from the singular elastic model in the upper panel demonstrate the progressive decay of the dilatational field and the increased separation between the leading dilatational field lobe and the shear Mach front with increased FN distance $\left(x_{2}\right)$ from the fault plane. Similar features are observed in the FP particle velocity waveforms depicted in the lower panel, which were obtained from five independent supershear experiments.

lationship, given by Eq. 32 between the rupture sliding speed $\dot{u}_{1}\left(x_{1}, 0^{+}\right)$and the FP velocity field component $\dot{u}_{1}\left(x_{1}, x_{2}\right)$ propagated by the shear Mach front. The results from supershear experiment (exp 0962) in Figure 20 are representative of a broader group of experiments, which were conducted and analyzed in a similar manner using the (FP1:FP2) laser vibrometer configuration.

The consecutive high-speed images in Figures 20(a,b) display the supershear rupture field as the propagating rupture tip and shear Mach front approached the on-fault and off-fault measurement stations positioned at $\left(-55,0^{+} \mathrm{mm}\right) \mathrm{mm}$ 

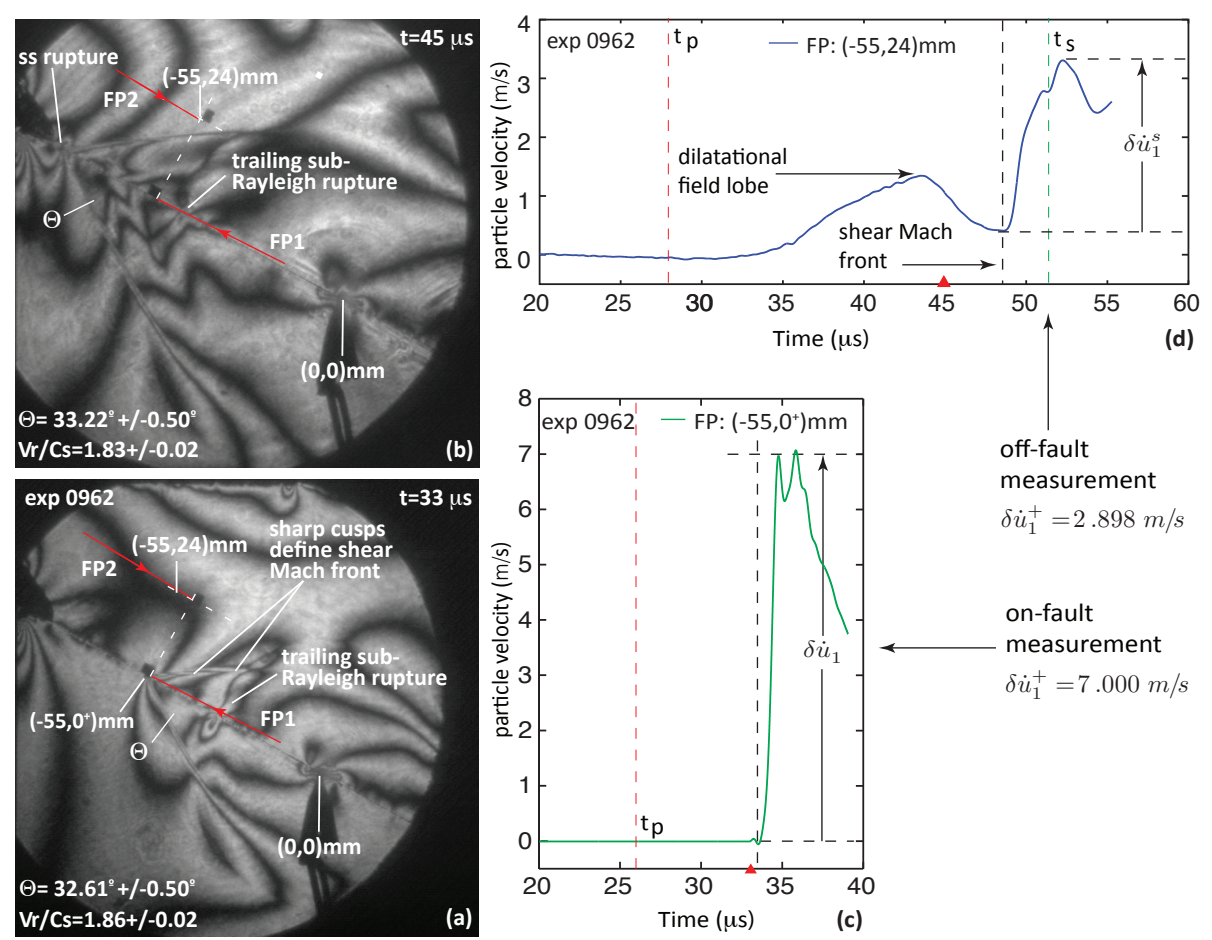

Figure 20: (a,b) Photoelastic image frames reveal the supershear rupture tip and the shear Mach front approaching the on-fault and off-fault measurement stations. (c,d) Truncated FP particle velocity waveforms recorded at the on-fault station $\left(-55,0^{+}\right) \mathrm{mm}$ and off-fault station $(-55,24) \mathrm{mm}$ as the rupture tip and shear Mach front swept the two locations.

and $(-55,24) \mathrm{mm}$. Figure $20 \mathrm{a}$, recorded at $t=33 \mu \mathrm{s}$, reveals the dilatational field lobe just as it began to sweep the on-fault measurement station $1190\left(-55,0^{+}\right) \mathrm{mm}$. Figure 20b, acquired at $t=45 \mu \mathrm{s}$, reveals the shear Mach front as it approached the off-fault station $(-55,24) \mathrm{mm}$. Mach angle measurements yielded a normalized rupture speed of $V_{r} / C_{s}=1.85$, which was consistent with the average rupture speed estimate obtained through a frame-by-frame analysis of the high-speed image sequence. Figures 20(c,d) display the on-fault and offfault FP particle velocity waveforms obtained in the experiment. Each waveform has been truncated in order to highlight the portion of the record attributed to the arrival of dilatational field and shear Mach front. The velocity jumps labeled $\delta \dot{u}_{1}^{+}$and $\delta \dot{u}_{1}^{s}$ in Figure 20 correspond to $\delta \dot{u}_{1}^{+}=\dot{u}_{1}^{\max }\left(-55,0^{+}\right)-\dot{u}_{1}^{\min }\left(-55,0^{+}\right)=$ $7.000 \mathrm{~m} / \mathrm{s}$ and $\delta \dot{u}_{1}^{s}=\left[\dot{u}_{1}^{s}(-55,24)\right]_{\max }-\left[\dot{u}_{1}^{s}(-55,24)\right]_{\min }=2.898 \mathrm{~m} / \mathrm{s}$, respectively. The measured on-fault and off-fault velocity jumps in (exp 0962) yield a ratio $\left(\delta \dot{u}_{1}^{s}\right)^{\text {meas }} /\left(\delta \dot{u}_{1}^{+}\right)^{\text {meas }}=0.414$. Application of Eq. 32 using the measured normalized rupture speed estimate of $V_{r} / C_{s}=1.85$ predicts a ratio of $\delta \dot{u}_{1}^{s} / \delta \dot{u}_{1}^{+}=0.416$. The measured value of 0.414 is thus found to be within $0.36 \%$ of the theoretically predicted value.

1205 Figure 21 summarizes the results obtained from 10 independent supershear 


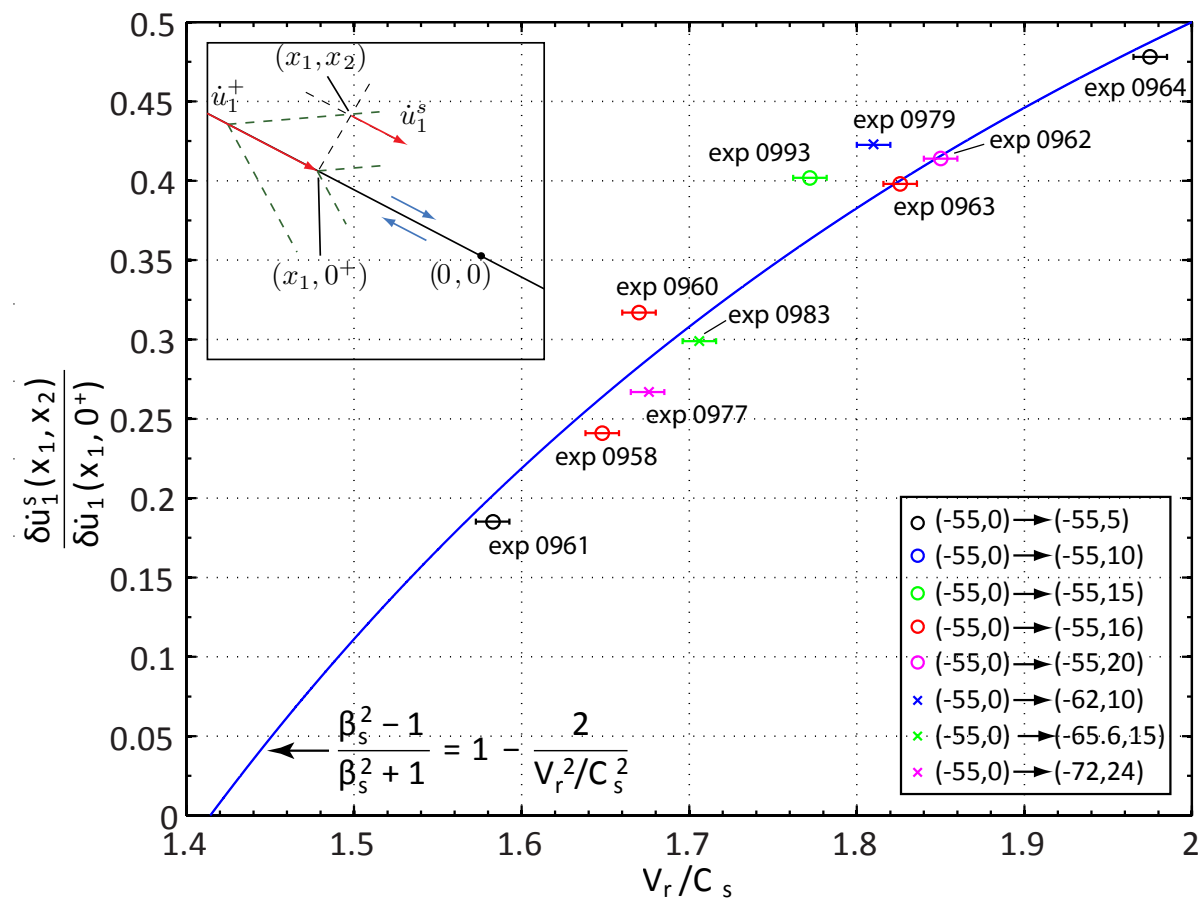

Figure 21: Plot of the measured ratio $\dot{u}_{1}^{s} / \dot{u}_{1}\left(x, 0^{+}\right)$obtained from 10 independent supershear experimental records plotted against the normalized wave speed $V_{r} / C_{s}$.

rupture experiments, which were analyzed in a similar manner. The inset in the upper left corner of the figure depicts the (FP1:FP2) laser vibrometer configuration, which simultaneously monitored the FP velocity component at the on-fault and off-fault field points. The solid blue curve corresponds to the scaling factor $\left(r_{s}\right)$, plotted versus the normalized rupture speed $\left(\sqrt{2}<V_{r} / C_{s}<2\right)$ in accordance with Eq. 32. The experimental estimates $\left(r_{s}^{\text {meas }}\right)$ represented by the colored circular symbols in the figure span across a considerable portion of the stable rupture speed regime. The symbol legend in the lower right corner provides a key to the coordinates of the on-fault and off-fault coordinates for each experiment. A high correlation is observed between the measured ratios obtained from the experiments and the theoretical scaling ratio given by Eq. 32. The largest error applies to exp 0993 and exp 0960, which deviate by $12 \%$ and $10.8 \%$ respectively, from the theoretical value. The larger deviation observed in these two experiments is attributed to the previously noted near field 1220 disturbance. We also note the range of $\mathrm{FN}$ distances over which the tests were conducted and how the results appear to be independent of the FN distance $\left(x_{2}\right)$. 


\section{Conclusions}

Spatiotemporal field properties and particle velocity waveform signatures of mode II sub-Rayleigh and supershear ruptures have been theoretically examined and experimentally verified. We have appealed to singular and nonsingular 2D steady state dynamic rupture solutions (Freund, 1979, Dunham and Archuleta, 2005) and gleaned unique scaling relationships between the FN and FP components of the shear and dilatational fields radiated by a supershear rupture. Near field particle velocity measurements were analyzed and interpreted within the context of these $2 \mathrm{D}$ steady-state theories.

The following properties of sub-Rayleigh rupture velocity fields were identified and experimentally verified by LEQ experiments using the (FN:FP) and (FN1:FN2) vibrometer probe configurations: (1) Sub-Rayleigh ruptures prop-

1235 agate with stable rupture speeds, which asymptotically approach the Rayleigh wave speed, i.e., $V_{r}<C_{R}$. (2) The particle velocity waveforms of a sub-Rayleigh rupture are distinguished by a $\mathrm{FN}$ component $\left(\dot{u}_{2}(t)\right)$, which always dominates in magnitude over the FP component $\left(\dot{u}_{1}(t)\right)$. (3) A strong correspondence was demonstrated between the predicted profile and polarity of synthetic particle velocity waveform profiles and particle velocity records corresponding subRayleigh ruptures observed in LEQ experiments. (4) The amplitude decay of the FN velocity field was experimentally investigated using the (FN1:FN2) vibrometer configuration. Measurements exposed the spatial extent of the rupture process zone and reveal an amplitude decay, which was reasonably well modeled 1245 by a $1 / \sqrt{r}$ curve fit for field points extending from the outer boundary of the rupture process zone out to a FN distance $x_{2}=10 \mathrm{~mm}$ from the fault plane.

The following properties of supershear rupture velocity fields were identified and experimentally verified by LEQ experiments using the (FN:FP) and (FP1:FP2) vibrometer probe configurations: (1) A concentrated dilatational field lobe was identified, which circulates about the supershear rupture and extends out well beyond the region bounded by the shear Mach fronts. The dilatational field lobe induces a velocity disturbance in the FP and FN waveforms, characterized by a dominant FP component $\left(\dot{u}_{1}^{d}\right)$, which always arrives in advance of the shear Mach front. (2) The dilatational field $\left(\dot{u}^{d}\right)$ contribution is identified in supershear particle velocity records and observed to decay rapidly with increased FN distance, albeit more slowly than the rate of decay of a sub-Rayleigh rupture field. (3) The shear and dilatational field contributions of a supershear rupture field are predicted to separate rapidly with increased distance from the fault plane. The effect is repeatedly observed in LEQ experi1260 ments and exploited to probe the particle velocity field components propagated by the shear Mach front. (4) Motion propagated by the shear Mach front is characterized by a FP component $\left(\dot{u}_{1}^{s}(t)\right)$, which dominates in magnitude over the FN component $\left(\dot{u}_{2}^{s}(t)\right)$ in the stable rupture speed regime $\left(\sqrt{2} C_{s} \leq V_{r} \leq C_{d}\right)$. (5) The ratio between the particle velocity jumps propagated by the shear Mach 1265 front was shown to observe the fundamental relationship $\frac{\delta \dot{u}_{1}^{s}}{\delta \dot{u}_{2}^{s}}=\beta_{s} \operatorname{sgn}\left(\xi_{2}\right)$. (6) A peculiar circumstance arises when $V_{r}=\sqrt{2} C_{s}$ whereby the shear Mach front 
is predicted to vanish. The effect was observed and analyzed during the investigation of a supershear daughter crack. and Archuleta, 2005) was also discussed and examined. The non-singular model recovers spatiotemporal field properties and scaling relations predicted by singular elastic theory. The solution given by (Dunham and Archuleta, 2005) applies equally well to crack-like ruptures observed in LEQ experiments by considering the limiting case of a very long pulse length. New scaling relationships were

1275 gleaned from the non-singular solution, which relate the supershear rupture sliding speed, measured at the fault plane, to the FP components of the shear and dilatational fields radiated away from the fault plane. In particular, the speed-dependent kinematic scaling relationship given by $r_{s}=\frac{\dot{u}_{1}^{s}\left(x_{1}, 0^{+}\right)}{\dot{u}_{1}\left(x_{1}, 0^{+}\right)}=\frac{\beta_{s}^{2}-1}{\beta_{s}^{2}+1}$, was derived and experimentally verified using the (FP1:FP2) configuration.

\section{Acknowledgements}

The authors gratefully acknowledge the National Science Foundation for the research grant (award no. EAR-0911723), provided under the American Recovery and Reinvestment Act of 2009 (ARRA) (Public Law 111-5).

\section{References}

Aagaard, B. T., Heaton, T. H., Dec 2004. Near-source ground motions from simulations of sustained intersonic and supersonic fault ruptures. Bull. Seismol. Soc. Amer. 94, 2064-2078.

Aki, K., Richards, P., 1968. Seismic didplacements near a fault. J. Geophy. Res. $73,5359-5376$.

Aki, K., Richards, P. G., 2002. Quantitative Seismology. University Science Books.

Andrews, D. J., 1976. Rupture velocity of plane strain shear cracks. J. Geophys. Res. 81 (B32), 5679-5689.

Barenblatt, G. I., 1962. The mathematical theory of equilibrium cracks in brittle fracture. Adv. Appl. Mech. 7 (2), 55-129.

Bhat, H. S., Dmowska, R., King, G. C. P., Klinger, Y., Rice, J. R., 2007. Offfault damage patterns due to supershear ruptures with application to the 2001 $M_{w}$ 8.1 Kokoxili (Kunlun) Tibet earthquake. J. Geophys. Res. B06301.

Bouchon, M., Bouin, M. P., Karabulut, H., Toksoz, M. N., Dietrich, M., Rosakis, A. J., Jul 2001. How fast is rupture during an earthquake? New insights from the 1999 Turkey earthquakes. Geophys. Res. Lett. 28, 2723-2726.

Bouchon, M., Karabulut, H., 2008. The aftershock signature of supershear earthquakes. Science 320 (5881), 1323. 
Bouchon, M., Toksoz, M. N., Karabulut, H., Bouin, M. P., Dietrich, M., Aktar, M., Edie, M., Feb 2002. Space and time evolution of rupture and faulting during the 1999 Izmit (Turkey) earthquake. Bull. Seismol. Soc. Amer. 92, $256-266$.

Bouchon, M., Vallee, M., Aug 2003. Observation of long supershear rupture during the magnitude 8.1 Kunlunshan earthquake. Science 301, 824-826.

Bouin, M., Bouchon, M., Karabulut, H., Aktar, M., 2004. Rupture process of the 1999 November 12 Duzce (Turkey) earthquake deduced from strong motion and global positioning system measurements. Geophys. J. Int, 159, 207-211.

Broberg, K. B., 1978. On transient sliding motion. Geophys. J. Roy. Astr. Soc $52,397-432$.

Broberg, K. B., 1989. The near-tip field at high crack velocities. Int. J. Fract. 39 (1), 1-13.

Broberg, K. B., Dec 1994. Intersonic bilateral slip. Geophys. J. Int. 119, 706-714.

Burridge, R., 1973. Admissible speeds for plane-strain self-similar shear cracks with friction but lacking cohesion. Geophys. J. Roy. Astron. Soc 35, 439-455.

${ }_{1320}$ Burridge, R., Conn, G., Freund, L. B., 1979. Stability Of a rapid mode-II shear crack with finite cohesive traction. J. Geophys. Res 84, 2210-2222.

Craggs, J., 1960. On the propagation of a crack in an elastic-brittle material. J. Mech. Phys. Solids 8, $66-75$.

Dunham, E. M., Archuleta, R. J., Dec 2004. Evidence for a supershear transient during the 2002 Denali fault earthquake. Bull. Seismol. Soc. Amer. 94, S256S268.

Dunham, E. M., Archuleta, R. J., Feb 2005. Near-source ground motion from steady state dynamic rupture pulses. Geophys. Res. Lett. 32 .

Dunham, E. M., Bhat, H. S., 2008. Attenuation of radiated ground motion and stresses from three-dimensional supershear ruptures. J. Geophys. Res. 113 (B08319).

Dunham, E. M., Favreau, P., Carlson, J. M., Mar 2003. A supershear transition mechanism for cracks. Science 299, 1557-1559.

Ellsworth, W. L., Celebi, M., Evans, J. R., Jensen, E. G., Kayen, R., Metz, M. C. Nyman, D. J., Roddick, J., P., S., Stephens, C. D., 2004. Near-field ground motion of the 2002 Denali fault, Alaska, earthquake recorded at pump station 10. Earthquake Spectra 20 (3), 597-615.

Fossum, A. F., Freund, L. B., 1975. Nonuniformly moving shear crack model of a shallow focus earthquake mechanism. J. Geophys. Res. 80 (B23), 3343-3347. 


\section{Res 84 (B5), 2199-2209.}

Freund, L. B., 1998. Dynamic Fracture Mechanics. Cambridge University Press, Cambridge.

F.X.Passelegue, Schubnel, A., Nielsen, S., Bhat, H., Madariaga, R., 2013. From Sub-Rayleigh to Supershear Ruptures During Stick-Slip Experiments on Crustal Rocks. Science 340 (6137), 1208-1211.

Gabuchian, V., Rosakis, A. J., Lapusta, N., Oglesby, D., 2014. Experimental investigation of strong ground motion due to thrust fault earthquakes. JGR 119 (2), $1316-1336$.

Graff, K. F., 1991. Wave Motion in Elastic Solids. Dover Publications, Inc.,.

Ida, Y., 1972. Cohesive force across tip of a longitudinal-shear crack and Griffiths specific surface-energy. J. Geophys. Res 77, 3796-3805.

Kostrov, B., 1964. Self similar problems of propagation of shear cracks. Appl. Math, Mech. 28, $889-898$.

Lambros, J., Rosakis, A. J., Feb 1995. Shear dominated transonic interfacial crack-growth in a bimaterial. 1. Experimental-observations. J. Mech. Phys. Solids 43, 169-188.

Latour, S., Gallot, T., Catheline, S., Voisin, C., Renard, F., Larose, E., Campillo, M., 2011. Ultrafast ultrasonic imaging of dynamic sliding friction in soft solids: The slow slip and the super-shear regimes. Europhysics Letters 96 (59003), 1-6.

Liu, C., Rosakis, A., 1994. On the higher order aymptotic analysis of a nonuniformly propagating dynamic crack along an arbirary path. Jounal of Elasticity $35(27-60)$.

Liu, Y., Lapusta, N., 2008. Transition of mode II cracks from sub-Rayleigh to intersonic speeds in the presence of favorable heterogeneity. J. Mech. Phys. Solids 56, 25-50.

Mello, M., Bhat, H., Rosakis, A., Kanamori, H., 2010. Identifying the unique ground motion signatures of supershear earthquakes: Theory and experiments. Tectonophysics 493 (3-4), 297-326.

Mello, M., Bhat, H., Rosakis, A., Kanamori, H., 2014. Reproducing the supershear portion of the 2002 Denali earthquake rupture in laboratory. Earth and Planetary Science Letters 387, $89-96$.

Palmer, A. C., Rice, J. R., 1973. Growth of slip surfaces in progressive failure ${ }_{1375}$ of over-consolidated clay. Proc. R. Soc. London. Ser. A-Math. Phys. Eng. Sci. $332,527-548$. 
Robinson, D. P., Brough, C., Das, S., Aug 2006. The $M_{w}$ 7.8, 2001 Kunlunshan earthquake: Extreme rupture speed variability and effect of fault geometry. J. Geophys. Res. 111 (B8).

1380 Rosakis, A. J., Xia, K. W., Lykotrafitis, G., Kanamori, H., 2007. Treatise in Geophysics. Vol. 4. Elsevier, Ch. Dynamic shear rupture in frictional interfaces: speeds, directionality and modes, pp. 153-192.

Samudrala, O., Huang, Y., Rosakis, A. J., Jun 2002a. Subsonic and intersonic mode II crack propagation with a rate-dependent cohesive zone. J. Mech. Phys. Solids 50, 1231-1268.

Samudrala, O., Huang, Y., Rosakis, A. J., Aug 2002b. Subsonic and intersonic shear rupture of weak planes with a velocity weakening cohesive zone. J. Geophys. Res. 107 (B8).

Svetlizky, I., Fineberg, J., 2014. Classical Shear Cracks Drive the Onset of Dry Frictional Motion. Nature 509, 205-208.

Xia, K. W., Rosakis, A. J., Kanamori, H., Mar 2004. Laboratory earthquakes: The sub-Rayleigh-to-supershear rupture transition. Science 303 (1859-1861), 1859-1861.

Xia, K. W., Rosakis, A. J., Kanamori, H., May 2005. Supershear and subRayleigh-to-supershear transition observed in laboratory earthquake experiments. Exp. Tech. 29, 63-66. 